\title{
Genome-resolved metagenome and metatranscriptome analyses of thermophilic composting reveal key bacterial players and their metabolic interactions
}

\author{
Lucas Palma Perez Braga ${ }^{1 \dagger}$, Roberta Verciano Pereira ${ }^{1 \dagger}$, Layla Farage Martins ${ }^{1 \dagger}$, Livia Maria Silva Moura ${ }^{1,2 \dagger}$,
} Fabio Beltrame Sanchez ${ }^{1,2}$, José Salvatore Leister Patané ${ }^{3}$, Aline Maria da Silva ${ }^{1 *}$ and João Carlos Setubal ${ }^{1 *}$

\begin{abstract}
Background: Composting is an important technique for environment-friendly degradation of organic material, and is a microbe-driven process. Previous metagenomic studies of composting have presented a general description of the taxonomic and functional diversity of its microbial populations, but they have lacked more specific information on the key organisms that are active during the process.

Results: Here we present and analyze 60 mostly high-quality metagenome-assembled genomes (MAGs) recovered from time-series samples of two thermophilic composting cells, of which 47 are potentially new bacterial species; 24 of those did not have any hits in two public MAG datasets at the $95 \%$ average nucleotide identity level. Analyses of gene content and expressed functions based on metatranscriptome data for one of the cells grouped the MAGs in three clusters along the 99-day composting process. By applying metabolic modeling methods, we were able to predict metabolic dependencies between MAGs. These models indicate the importance of coadjuvant bacteria that do not carry out lignocellulose degradation but may contribute to the management of reactive oxygen species and with enzymes that increase bioenergetic efficiency in composting, such as hydrogenases and $\mathrm{N}_{2} \mathrm{O}$ reductase. Strong metabolic dependencies predicted between MAGs revealed key interactions relying on exchange of $\mathrm{H}^{+}, \mathrm{NH}_{3}, \mathrm{O}_{2}$ and $\mathrm{CO}_{2}$, as well as glucose, glutamate, succinate, fumarate and others, highlighting the importance of functional stratification and syntrophic interactions during biomass conversion. Our model includes 22 out of 49 MAGs recovered from one composting cell data. Based on this model we highlight that Rhodothermus marinus, Thermobispora bispora and a novel Gammaproteobacterium are dominant players in chemolithotrophic metabolism and cross-feeding interactions.
\end{abstract}

* Correspondence: almsilva@iq.usp.br; setubal@iq.usp.br

† Lucas Palma Perez Braga, Roberta Verciano Pereira, Layla Farage Martins and Livia Maria Silva Moura contributed equally to this work.

'Departamento de Bioquímica, Instituto de Química, Universidade de São Paulo, São Paulo, Brazil

Full list of author information is available at the end of the article

C C The Author(s). 2021 Open Access This article is licensed under a Creative Commons Attribution 4.0 International License, which permits use, sharing, adaptation, distribution and reproduction in any medium or format, as long as you give appropriate credit to the original author(s) and the source, provide a link to the Creative Commons licence, and indicate if changes were made. The images or other third party material in this article are included in the article's Creative Commons licence, unless indicated otherwise in a credit line to the material. If material is not included in the article's Creative Commons licence and your intended use is not permitted by statutory regulation or exceeds the permitted use, you will need to obtain permission directly from the copyright holder. To view a copy of this licence, visit http://creativecommons.org/licenses/by/4.0/ The Creative Commons Public Domain Dedication waiver (http://creativecommons.org/publicdomain/zero/1.0/) applies to the data made available in this article, unless otherwise stated in a credit line to the data. 
Conclusions: The results obtained expand our knowledge of the taxonomic and functional diversity of composting bacteria and provide a model of their dynamic metabolic interactions.

Keywords: Metagenome-assembled genome, Microbiome, Biomass degradation, Rhodothermus marinus, Thermobispora bispora

\section{Background}

Thermophilic composting is carried out by microbial communities that are able to thrive in this harsh environment $[1,2]$. Recent studies have demonstrated that composting microbiomes comprise an enormous diversity of mesophilic and thermophilic microorganisms depending on the method and conditions as well as the stage of the composting process [1-5]. Composting microbes present a remarkable metabolic flexibility and are efficient in breaking down complex organic matter such as lignocellulosic biomass $[1,4,6]$.

Lignocellulosic biomass is composed by different biopolymers: cellulose (25-55\%), hemicellulose (19-40\%), lignin (18-35\%), and smaller fractions of pectin and minerals. Therefore, a diverse set of enzymes is required for effective saccharification [7]. Microbial dynamics during lignocellulose breakdown seems to be heavily dependent on syntrophic interactions [8]. The microbial populations need to share the burden of enzymatic production; and sharing metabolites reduces the negative feedback effect of intermediate metabolite accumulation [9]. Syntrophic interactions can involve opportunistic microbes in biomass degrading systems, which are bacteria that do not express or very often lack the required enzymes for biomass degradation, but constitute one important portion of the microbial community, being referred to as 'sugar cheaters' [8].

The composting microbiome is considered a valuable microbial resource for biomass degradation, with potential for contributing to a number of biotechnological applications besides its remarkable activities on soil bioremediation and suppressiveness against plant diseases $[2,6,10,11]$. In spite of this potential, knowledge on how to control and explore those microbes and their functions remains encrypted within their genomes and the multiple combinations of metabolic pathways that they can activate $[6,8,12]$. Research on composting microbes has focused mainly on enriched cultures $[8,13$, 14] or taxonomic biodiversity assessments based on $16 \mathrm{~S}$ rRNA gene amplicon sequencing data $[3,5]$. Yet, these methods cannot fully assess microbial functional diversity and metabolic activity.

Shotgun metagenomic sequencing has helped to reveal the diversity of microbial communities in natural habitats [15] and in engineered environments such as composting [1, 16] or sludge digesters [17]. New methods and computational tools now allow the recovery of metagenome-assembled genomes (MAGs) from complex ecosystems [18-23]. The study of MAGs from microorganisms in a given environment can provide detailed taxonomical and functional diversity information, and therefore has the potential to allow a better understanding of their ecological context and metabolic arsenal [22, 24].

Here we present an analysis of MAGs obtained from time-series samples of a thermophilic composting process. These datasets have been analyzed previously, but not from a MAG perspective [1]. Our aim was to obtain a detailed view of the microbial populations active in a composting process and to determine their metabolic interactions, thus advancing on our previous work [1]. We used the collection of genomes recovered to build a framework for the temporal dynamics of microbial molecular processes during composting. Using this framework as a reference, we built genome-scale metabolic models for predicting syntrophic interactions and the more frequently exchanged compounds.

\section{Results}

\section{MAGs recovered from thermophilic composting}

We recovered a total of 11 and 49 MAGs (Metagenomeassembled genomes), respectively, from metagenomes of ZC3 and ZC4 composting samples (Table 1). All these 60 MAGs (Supplementary Table 1) meet the mediumquality requirement $(\geq 50 \%$ completeness and $\leq 10 \%$ contamination) of the MIMAG standard [25], with the exception of ZC3RG09, which had $10.87 \%$ contamination. Thirty-four MAGs meet the high-quality requirement ( $\geq 90 \%$ completeness and $\leq 5 \%$ contamination). The average number of contigs in these MAGs is 363.75 , with a minimum of 15 (ZC4RG10) and a maximum of 1655 (ZC4RG48) (Supplementary Table 1). The genome size of the 60 MAGs varies from $5.7 \mathrm{Mbp}$ (ZC4RG46) to $1.5 \mathrm{Mbp}$ (ZC4RG49) and their average \%GC is $64.21 \pm$ 8.78 (Supplementary Table 1). Pairwise comparisons (Supplementary Table 2) showed that six MAGs recovered from ZC3 metagenomes (ZC3RG05, ZC3RG06, ZC3RG07, ZC3RG08, ZC3RG09 and ZC3RG11) are highly similar (two-way ANI measure $\geq 99 \%$; DDH estimate $\geq 95 \%$ ) to six MAGs recovered from ZC4 metagenomes (ZC4RG21, ZC4RG10, ZC4RG11, ZC4RG09, ZC4RG06 and ZC4RG18, respectively); this level of similarity is what we call 'MAG redundancy' further down. 
Table 1 MAGs recovered from ZC3 and ZC4 thermophilic composting

\begin{tabular}{|c|c|c|c|}
\hline MAG & Taxonomy classification according to GTDB (Genome Taxonomy Database) & Species assignment & \# reads (DNA) \\
\hline ZC3RG01 & d_Bacteria;p_Bacteroidota;c_Bacteroidia;o_Bacteroidales; f F082;g_F082;s & - & 115463 \\
\hline * ZC3RG02 & d_Bacteria;p_Firmicutes_A;c_Clostridia;o_Oscillospirales;f_Oscillospiraceae;g_CAG-110;s & - & 81537 \\
\hline * ZC3RG03 & d_Bacteria;p_Firmicutes_A;c_Clostridia;o_Christensenellales;f_CAG-74;g_;s_ & - & 153378 \\
\hline ZC3RG04 & d_Bacteria;p_Bacteroidota;c_Bacteroidia;o_Bacteroidales;f_WCHB1-69;g_UBA $1756 ; \mathrm{s}$ & - & 244322 \\
\hline ZC3RG05 & d_Bacteria;p_Actinobacteriota;c_Actinobacteria;o__Streptosporangiales;f_Streptosporangiaceae;g_Thermobifida & Thermobifida fusca & 553516 \\
\hline * ZC3RG06 & d Bacteria;p Firmicutes G;c Limnochordia;o DTU080; $; \mathrm{g} ; \mathrm{s}$ & - & 484812 \\
\hline * ZC3RG07 & d Bacteria;p Firmicutes G;c Limnochordia;o Limnochordales; $\mathrm{f}$ Limnochordaceae;g Limnochorda;s & - & 365253 \\
\hline ZC3RG08 & d_Bacteria;p_Firmicutes;c_Bacilli_A;o_Thermoactinomycetales;f_Thermoactinomycetaceae;g Planifilum & Planifilum fulgidum & 298830 \\
\hline * ZC3RG09 & d_Bacteria;p_Firmicutes_G;c_Limnochordia;o_Limnochordales;f_ZCTH02-B6;g_ZCTH02-B6;s & - & 210189 \\
\hline * ZC3RG10 & d Bacteria;p Firmicutes_G;c Limnochordia;o Limnochordales; & - & 167600 \\
\hline ZC3RG11 & d Bacteria; $p$ Firmicutes E;c Thermaerobacteria;o Thermaerobacterales; $f ; g ; s$ & - & 97976 \\
\hline ZC4RG02 & d_Bacteria;p_Proteobacteria;c_Gammaproteobacteria;o_Xanthomonadales;f_Xanthomonadaceae;g_Pseudoxanthomonas;s & - & 476308 \\
\hline * ZC4RG03 & d_Bacteria;p_Firmicutes;c_Bacilli_A;o_Calditerricolales;f_Calditerricolaceae;g_Calditerricola;s_- & - & 1020829 \\
\hline ZC4RG04 & d_Bacteria;p_Actinobacteriota;c_Actinobacteria;o_Streptosporangiales;f_Streptosporangiaceae;g_Thermobispora & Thermobispora bispora & 1126372 \\
\hline ZC4RG05 & d_Bacteria;p_Firmicutes_G;c_Limnochordia;o_Limnochordales; $\mathrm{f}$ ZCTH $02-\mathrm{B} 6 ; \mathrm{g}$; & - & 1643496 \\
\hline * ZC4RG06 & d_Bacteria;p_Firmicutes_G;c_Limnochordia;o_Limnochordales;f_ZCTH02-B6;g_ZCTH02-B6;s & - & 1397340 \\
\hline * ZC4RG07 & d_Bacteria;p_Firmicutes;c_Bacilli_A;o_Bacillales_F;f_Bacillaceae_M;g_Bacillus_BB;s_GCA_001 1884825.1 & - & 431834 \\
\hline ZC4RG08 & d_Bacteria;p_Proteobacteria;c_Gammaproteobacteria;o_Pseudomonadales;f_Pseudomonadaceae;g_Pseudomonas_G & Pseudomonas thermotolerans & 257892 \\
\hline ZC4RG09 & d_Bacteria;p_Firmicutes;c_Bacilli_A;o_Thermoactinomycetales;f_Thermoactinomycetaceae;g_Planifilum & Planifilum fulgidum & 932805 \\
\hline * ZC4RG10 & d_Bacteria;p_Firmicutes_G;c Limnochordia;o_DTU080; $; \mathrm{g} ; \mathrm{s}$ & - & 1320702 \\
\hline * ZC4RG11 & d_Bacteria;p_Firmicutes_G;c_Limnochordia;o_Limnochordales; $;$ Limnochordaceae; $g$ Limnochorda;s & - & 397116 \\
\hline * ZC4RG12 & d_Bacteria;p_Firmicutes_A;c_Mahellia;o_Caldicoprobacterales;f_Caldicoprobacteraceae;g_Caldicoprobacter;s & - & 621385 \\
\hline ZC4RG15 & d_Bacteria;p_Firmicutes;c_Bacilli;o_Bacillales_A;f Planococcaceae;g_Ureibacillus;s & - & 290499 \\
\hline ZC4RG16 & d_Bacteria;p_Gemmatimonadota;c_Gemmatimonadetes;o SG8-23;f; ; _ ; & - & 1344615 \\
\hline ZC4RG17 & d Bacteria;p_Actinobacteriota;c Acidimicrobiia;o bin $76 ; \mathrm{f} ; \mathrm{g}$;s & - & 640885 \\
\hline ZC4RG18 & d_Bacteria;p_Firmicutes_E;c_Thermaerobacteria;o_Thermaerobacterales;f_;g_; & - & 476723 \\
\hline ZC4RG19 & d_Bacteria;p_Actinobacteriota;c_Acidimicrobiia;o_Microtrichales;f_UBA9382;g_; & - & 646656 \\
\hline * ZC4RG20 & d_Bacteria;p_Proteobacteria;c_Gammaproteobacteria;0_UBA6522;f_UBA6522;g_; & - & 640062 \\
\hline ZC4RG21 & d Bacteria; $p$ Actinobacteriota;c Actinobacteria;o Streptosporangiales $;$ Streptosporangiaceae; $g$ Thermobifida & Thermobifida fusca & 406751 \\
\hline ZC4RG22 & d Bacteria;p Acidobacteriota;c Luteitaleia;o Luteitaleales;f SCN-69-37;g SCN-69-37;s & - & 414892 \\
\hline * ZC4RG23 & d Bacteria;p Firmicutes E;c Symbiobacteria;o Symbiobacteriales;f Symbiobacteriaceae;g Symbiobacterium;s & - & 346328 \\
\hline ZC4RG24 & d Bacteria;p Proteobacteria;c Gammaproteobacteria;o Steroidobacterales;f Steroidobacteraceae;g 13-2-20CM-66-19;s & - & 401351 \\
\hline ZC4RG25 & d Bacteria;p Proteobacteria;c Alphaproteobacteria;o Rhizobiales;f Hyphomicrobiaceae;g ;s & - & 385716 \\
\hline ZC4RG26 & d Bacteria;p Chloroflexota; c Chloroflexia;o Thermomicrobiales; $\mathrm{f}$ Thermomicrobiaceae;g Sphaerobacter & Sphaerobacter thermophilus & 519449 \\
\hline ZC4RG27 & d_Bacteria;p_Proteobacteria;c_Alphaproteobacteria;o_Rhizobiales;f_Beijerinckiaceae;g_Chelatococcus;s & - & 238524 \\
\hline ZC4RG28 & d Bacteria;p Actinobacteriota;c Actinobacteria;o Streptosporangiales;f Streptosporangiaceae;g ;s & - & 745493 \\
\hline * ZC4RG29 & d_Bacteria;p_Bacteroidota;c_Bacteroidia;o_Cytophagales;f_Cyclobacteriaceae;g_;s_ & - & 304810 \\
\hline * ZC4RG30 & d_Bacteria;p_Proteobacteria;c Gammaproteobacteria;o_Steroidobacterales; $f$ Steroidobacteraceae; $\mathrm{g}$;s & - & 286066 \\
\hline ZC4RG31 & d Bacteria;p Proteobacteria;c Alphaproteobacteria;o Rhizobiales;f Hyphomicrobiaceae;g ;s & - & 341014 \\
\hline ZC4RG32 & d_Bacteria;p_Firmicutes_A;c_Mahellia;o_Caldicoprobacterales;f_Caldicoprobacteraceae;g_Caldicoprobacter & Caldicoprobacter oshimai & 270288 \\
\hline * ZC4RG33 & d_Bacteria;p_Proteobacteria;c_Alphaproteobacteria;o_Rhizobiales;f_Rhizobiaceae;g_Aquamicrobium;s_ & - & 231251 \\
\hline * ZC4RG34 & d_Bacteria;p_Firmicutes_A;c_Thermovenabulia;o_Thermovenabulales;f_Thermovenabulaceae;g_; & - & 199309 \\
\hline * ZC4RG35 & d_Bacteria;p_Actinobacteriota;c_Acidimicrobiia;o_bin $76 ; \mathrm{f}_{\mathrm{a}} ; \mathrm{g}$; & - & 140681 \\
\hline ZC4RG36 & d Bacteria;p Chloroflexota;c Anaerolineae;o SBR $1031 ; \mathrm{f}$ A4b;g ; & . & 343246 \\
\hline ZC4RG40 & d_Bacteria;p_Myхососcota;c_Myхососcia;o_Myxococcales;f_Vulgatibacteraceae;g_; & - & 392408 \\
\hline * ZC4RG41 & d Bacteria;p_Actinobacteriota;c Actinobacteria;o Actinomycetales;f Demequinaceae;g ;s & - & 122956 \\
\hline ZC4RG42 & d Bacteria;p_Proteobacteria;c Gammaproteobacteria;o Betaproteobacteriales;f SG8-39;g 2-12-FULL-64-23;s & - & 125698 \\
\hline ZC4RG43 & d_Bacteria;p_Actinobacteriota;c_Actinobacteria;o_Corynebacteriales;f_Corynebacteriaceae;g_Mycobacterium & Mycobacterium hassiacum & 226699 \\
\hline * ZC4RG44 & d_Bacteria;p_Firmicutes_E;c_Thermaerobacteria;o_Thermaerobacterales;f_Thermaerobacteraceae;g_Thermaerobacter;s_ & - & 259754 \\
\hline ZC4RG45 & d_Bacteria;p_Actinobacteriota;c_Actinobacteria;o_Corynebacteriales;f_Pseudonocardiaceae;g_Thermocrispum & Thermocrispum agreste & 198356 \\
\hline * ZC4RG46 & d Bacteria;p Myxococcota;c Polyangia;o Polyangiales;f NIC37A-2;g ;s & - & 309368 \\
\hline * ZC4RG47 & d_Bacteria;p_Actinobacteriota;c_Actinobacteria;o_Corynebacteriales;f_Micromonosporaceae;g_Micromonospora;s_ & - & 216425 \\
\hline * ZC4RG48 & d_Bacteria;p_Chloroflexota;c_Chloroflexia;o_Chloroflexales;f_Roseiflexaceae;g_;s_ & - & 216650 \\
\hline ZC4RG49 & d Bacteria; $p$ Firmicutes A;c Clostridia;o Oscillospirales; $f$ Ethanoligenenaceae;g $\overline{\text { Ruminiclostridium D }}$ & [Clostridium] cellulosi & 73802 \\
\hline
\end{tabular}

* MAGs for which there were no hits in the GEM catalog

These MAGs may represent genomes from different strains from the same bacterial species.

Mapping of ZC4 metagenome reads to ZC4 MAGs shows that the 49 ZC4 MAGs account for $21.8 \%$ of all $\mathrm{ZC} 4$ reads (the number of $\mathrm{ZC} 4$ metagenome reads per MAG is shown in the last column of Table 1; adding that column results in $24,539,668$ reads. The total number of ZC4 reads is 112,736,134 [1]). A similar calculation for ZC3 shows that the 11 ZC3 MAGs account for $29.6 \%$ of all reads in those samples.

\section{Taxonomic assignments}

The 60 recovered MAGs were assigned to six different phyla: Acidobacteriota, Actinobacteriota, Bacteroidota, Chloroflexota, Firmicutes and Proteobacteria (Table 1) according to GTDB [26]. At the order level there is remarkable diversity, with 32 different orders represented by these MAGs. The most frequent order was Limnochordales (6 MAGs). Most of the ZC3 and ZC4 MAGs seem to be novel: there are eight MAGs for which no family could be assigned, 18 MAGs for which no genus could be assigned, and 17 MAGs for which no species could be assigned; this takes into consideration the "redundancy" in MAGs between ZC3 and ZC4.

Thirteen MAGs could be assigned to 11 species (Table 1) for which there is at least one isolate genome publicly available in the NCBI RefSeq repository (Supplementary Table 3). Pairwise genome comparisons showed that in all these cases the two-way ANI measure was at least $98 \%$ and the DDH estimate was at least $87 \%$ (Supplementary Table 3), strongly suggesting that the assignments are correct and confirming that the recovered 
MAGs are of high quality. Several of the 11 species to which these 13 MAGs were assigned are known as thermophilic bacteria: Thermobifida fusca, Thermobispora bispora, Pseudomonas thermotolerans, Rhodothermus marinus, and Planifilum fulgidum. Except for Mycobacterium hassiacum, which has been isolated from human samples, the other 10 species have been found in environments related to biomass degradation, such as compost, decaying wood, and animal feces (Supplementary Table 4).

\section{ZC3 and ZC4 MAGs in other environments}

We checked for the presence of our 60 MAGs in two publicly available MAG datasets [22, 23]. The recovery of the "same" genome from different environments lends additional confidence to our MAG recovery process. Among the 910 MAGs from the Asian soil, plant-based compost, and leafy greens phytobiomes [23] we found no relevant hits (i.e., ANI was less than $85 \%$ ). We did find hits (ANI $\geq 95 \%$ ) for 30 of our ZC3/ZC4 60 MAGs in the Genomes from the Earth's Microbiomes (GEM)

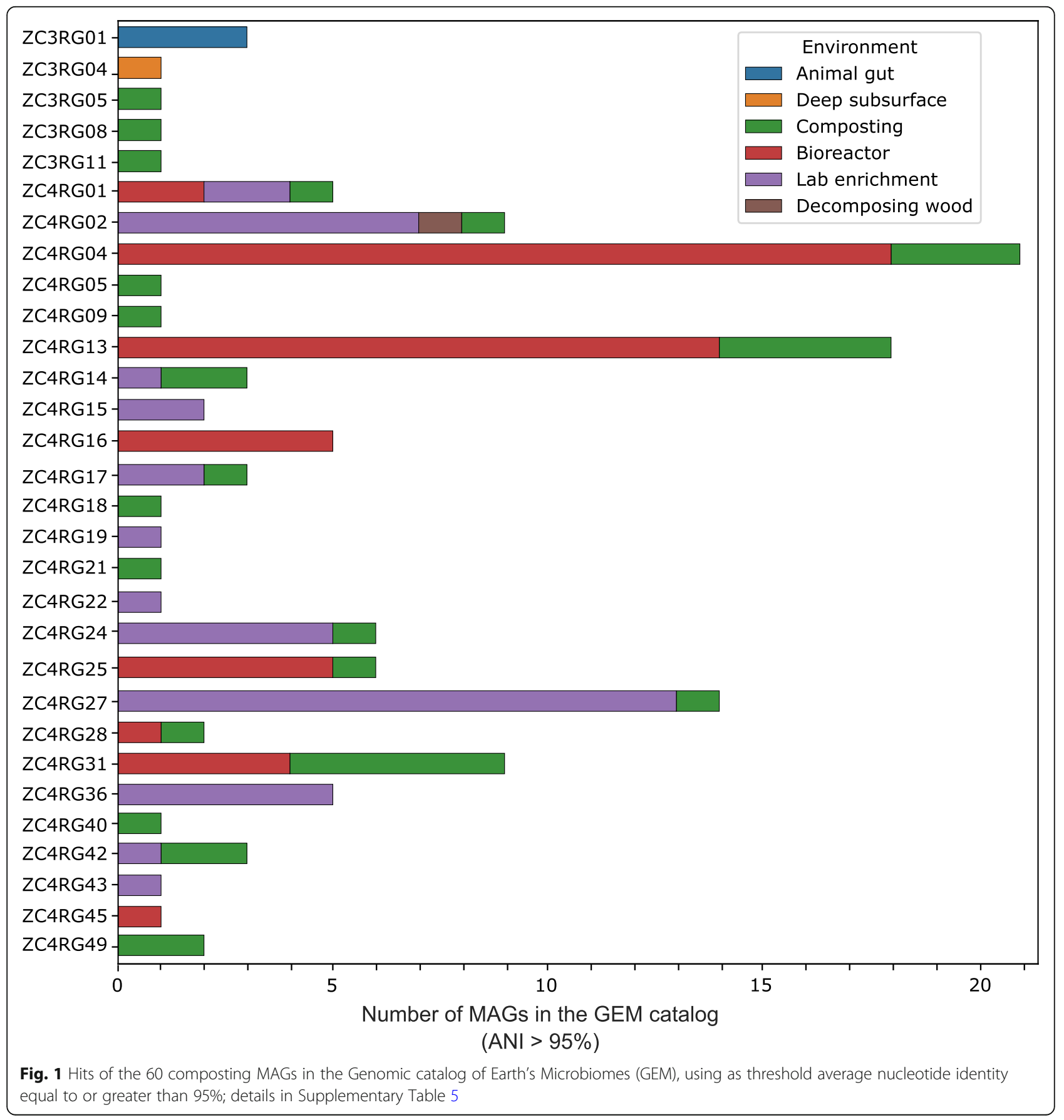


catalog [22] (Fig. 1, Supplementary Table 5). Genomes similar to these 30 MAGs were mostly recovered from environments associated with biomass-degradation, including cellulose-adapted laboratory enrichments and composting environments, as well as animal (capybara and moose guts), deep subsurface shale carbon reservoir, and bioreactor metagenomes (Fig. 1), suggesting that these bacterial populations are highly specific to biomassdegrading environments (Fig. 1, Supplementary Table 5). ZC4RG04 (Thermobispora bispora) and ZC4RG13 (Rhodothermus marinus) were the MAGs with most hits in the GEM catalog (21 and 18 hits, respectively). With this analysis we observed that some MAGs co-occurring in the Zoo composting were also co-occurring in other environments. For instance, GEM MAGs corresponding to ZC4RG05 (Limnochordales), ZC3RG11/ZC4RG18 (Thermaerobacterales), and ZC3RG08/ZC4RG09 (Planifilum fulgidum), were also recovered from a steer manure compost metagenome dataset (Supplementary Table 5).

Additional comparisons (not shown) revealed that ZC4RG01 (Caldibacillus debilis) and ZC3RG09/ZC4RG06 are similar (ANI $\geq 99 \%$ ), respectively, to genomes BZ5 and BZ6, which are two MAGs recovered from a thermophilic compost-derived consortium named ZCTH02 [13]. The composting facility from which this consortium was obtained is the same that provided the 60 MAGs here studied. ZC3RG09/ZC4RG06, ZC3RG10, and ZC4RG05 have been assigned by the GTDB group to a provisionally named family called ZCTH02-B6, in the order Limnochordales (Table 1).

Based on the results above, in our MAG dataset there are 24 nonredundant MAGs that have no species assigned and have no hits in the two MAG catalogs against which we searched, and therefore represent truly novel contributions to known MAG diversity.

\section{Functional analysis of composting MAGs}

For functional analysis, we have focused on ZC4 MAGs. Our rationale was that only for ZC4 samples do we have metatranscriptome data. These RNA-seq datasets were obtained for eight time-series samples (days 1, 3, 7, 15, 30, 64, 78 and 99 of composting) as previously reported [1]. The temperature of ZC4 composting cell at the day of sample collection varied from $66.2^{\circ} \mathrm{C}$ (day 1) to $47.8^{\circ} \mathrm{C}$ (day 99), being around $65-70^{\circ} \mathrm{C}$ most of the time, as detailed in Table 1 of Antunes et al. [1]. The relative abundance in transcripts per kilobase million reads (TPM) for each one of the 49 MAGs over time showed that all of them were transcriptionally active (Supplementary Table 6).

\section{Lignocellulose degradation}

Biomass degrading capabilities in MAGs were analyzed based on COG (Clusters of Orthologous Groups) assignments (Supplementary Fig. 1) and CAZy annotations
(Supplementary Tables 7 and 8; Fig. 2) of their respective genes. Among the 49 ZC4 MAGs, 26 encode more than 100 genes classified as CAZymes (Supplementary Table 7). Out of these, 14 MAGs encode at least 40 genes classified as GHs (Glycoside Hydrolases) (Fig. 2a; Supplementary Table 7). Several cellulases (GH5, GH6, GH9 and GH45), endohemicellulases (GH8, GH10, GH11, GH12, GH26, GH28 and GH53), debranching (GH51, GH62, GH67 and GH78) and oligosaccharidedegrading enzymes (GH1, GH2, GH3, GH29, GH35, GH38, GH39, GH42 and GH43) were annotated in these MAGs (Fig. 2b and c). The categorization of GHs just presented follows the categorization of the CAZyme database [27].

Regarding Auxiliary Activities (AA), ZC4RG20 (Gammaproteobacteria), ZC4RG33 (Aquamicrobium), ZC4RG43 (Mycobacterium hassiacum), and ZC4RG45 (Thermocrispum agreste) present at least 15 genes classified as AA (Supplementary Table 7). ZC4RG45 contains the highest diversity of AA genes (Supplementary Table 8). Members of the AA1 family, which perform lignin degradation efficiently, were only found in ZC4RG08 (Pseudomonas thermotolerans) (Supplementary Table 8). ZC4RG21 (Thermobifida fusca), ZC4RG04 (Thermobispora bispora), ZC4RG28 (Streptosporangiaceae), ZC4RG45, and ZC4RG47 (Micromonospora) were the only ones containing genes classified in the AA10 family (lytic polysaccharide monooxygenases) (Supplementary Table 8), members of which are capable of directly targeting cellulose for oxidative cleavage of the glucose chains.

As stated above, we have strong evidence that each ZC4 MAG here analyzed was transcriptionally active during composting. We checked the expression of genes related to lignocellulose degradation and determined that all CAZymes mentioned had corresponding transcripts in the ZC4 metatranscriptome dataset, and their abundance varies over time (Fig. 3 and Supplementary Table 9).

\section{Secondary metabolites}

Several coding sequences classified as secondary metabolite genes (such as siderophores, bacteriocins, sacpeptides, betalactones, lassopeptides, and type I, II and III polyketides) were found in MAGs (Fig. 4 and Supplementary Table 10). MAGs with at least 100 genes annotated as secondary metabolites were: ZC4RG04 (Thermobispora bispora), 121 genes; ZC4RG21 (Thermobifida fusca), 105 genes; ZC4RG22 (Luteitaleales), 115 genes; and ZC4RG39 (Steroidobacteraceae), 100 genes. (The MAG with the fifth largest repertoire of secondary metabolite genes had 58.) Transcripts for secondary metabolite genes were 


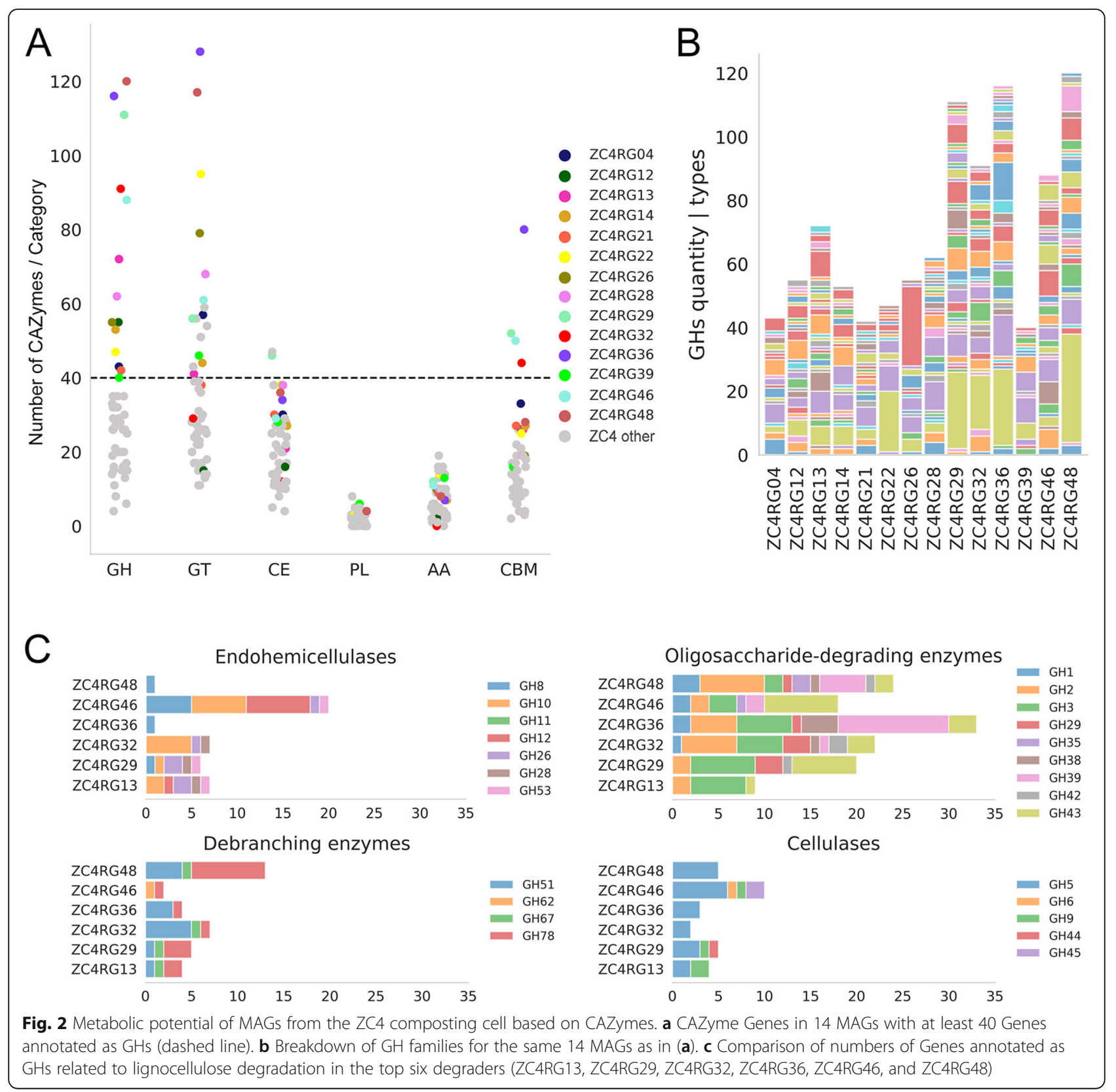

generally more abundant at the beginning of the composting process (days 1,3 , and 7) than in the later stages (days 78 and 99) (Supplementary Table 10). The range in TPM values greater than zero per gene per sample day was quite large: from near zero to 2162 , with the vast majority $(95.46 \%)$ being less than 50 . We can therefore define a highly expressed (HE) gene as secondary metabolism gene with at least 50 TPM in a sample day. Using this definition, the following MAGs stand out: ZC4RG04 (T. bispora) had $34 \mathrm{HE}$ genes on days 1 and 3, the most of any MAG, being the activity level on other days much smaller;
ZC4RG03 (Calditerricola) and ZC4RG07 (Bacillus) had the most HE genes in days $1,3,7,15$, and 64: 47 for ZC4RG03 and 32 for ZC4RG07. The peak day, however, was different: for ZC4RG07 it was on day 3 (13 HE genes), and for ZC4RG03 it was on day 15 (14 HE genes). For ZC4RG03 moreover, expression of any secondary metabolite gene was essentially zero on days 30, 78, and 99. ZC4RG21 ( $T$. fusca) was by far the MAG with most HE genes on day 30 (15 HE genes). The gene with the maximum TPM value (2162.5) belongs to ZC4RG03 (Calditerricola) and was annotated as a plantaricin $\mathrm{C}$ family lantibiotic (locus 


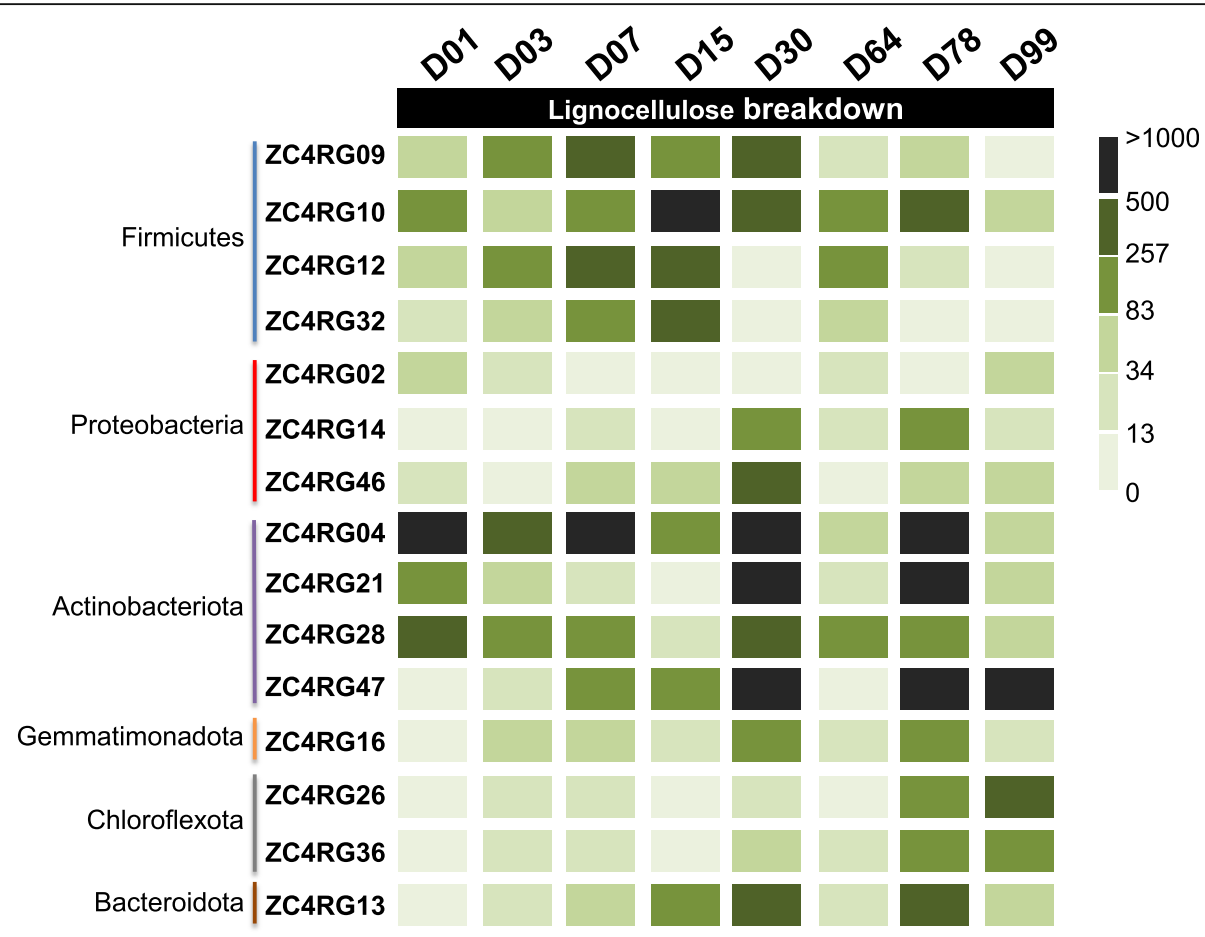

Fig. 3 Heatmap representing the abundance of transcripts associated with genes annotated with functions related to lignocellulose degradation in the indicated MAGs. The scale in shades of green is based on relative abundance (Transcripts per kilobase Million Reads) obtained from data shown in Supplementary Table 9

tag C0P64_01260). It is interesting to note that the two adjacent and upstream genes were also annotated as secondary metabolite genes (lantipeptide synthetase LanM and bacitracin ABC transporter ATP-binding protein), and they were also HE.

\section{Antibiotic resistance genes}

Antibiotic resistance genes (ARGs) were observed mainly in MAGs from Actinobacteriota and Proteobacteria phyla (Supplementary Table 11). ZC4RG08 (Pseudomonas thermotolerans) has the largest number

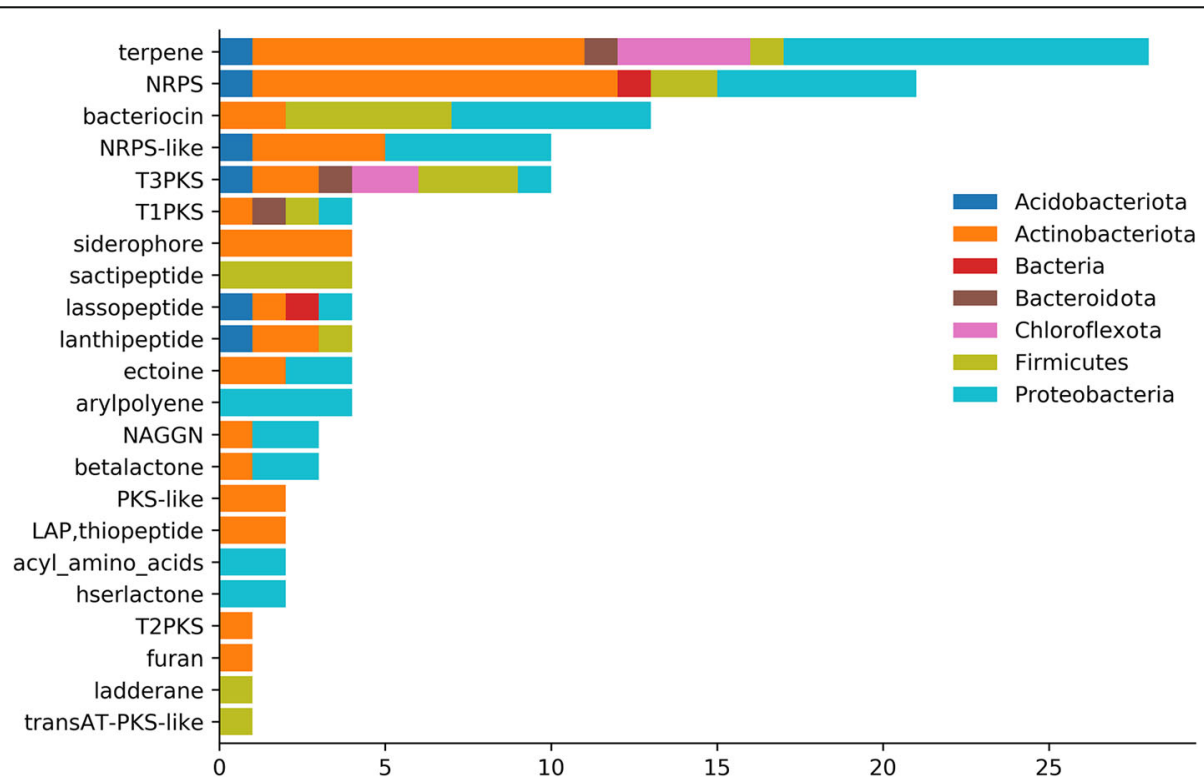

Fig. 4 Secondary metabolite cluster types detected among the ZC4 MAGs. The X-axis represents the number of clusters detected. The colors in the bars correspond to phylum assignment of MAGs 
of expressed ARGs, with 12 genes encoding multidrug efflux pumps (MuxABC-OpmB, MexAB-OprM, MexEFOprN, MexWV, and MexJK). The MAG that is ranked second in terms of number of expressed ARGs is ZC4RG43 (Mycobacterium hassiacum), with seven genes; all other MAGs express five or fewer ARGs (Supplementary Table 11). Among frequently detected ARGs, two genes may be remarked, because each of them is expressed by seven MAGs (out of 15): rpoB2, which encodes a rifampicin-refractory beta-subunit of RNA polymerase [28], and $m t r A$, which encodes the MtrCDE multidrug efflux pump transcriptional activator [29] (Supplementary Table 11). Overall, transcripts of ARGs were more abundant in days $1,3,30$ and 78 of the composting process (Supplementary Table 11).

\section{Aerobic and anaerobic respiration strategies}

The analysis of oxygen metabolism indicates that 45 out 49 bacteria from which MAGs were obtained are aerobes
(Table 2). All the oxidase genes annotated in the ZC4 MAGs are predicted to be active and their transcript abundance variation over time shows a slight decrease after day 7, with an increase following day 64 (immediately after the turning procedure of the composting cell) (Supplementary Fig. 2). Evidence of oxidases and aerobic metabolism was not detected in MAGs ZC4RG12 (Caldicoprobacter), ZC4RG32 (C. oshimai), ZC4RG34 (Thermovenabulaceae), and ZC4RG49 ([Clostridium] cellulosi), thereby indicating a metabolism strictly anaerobic. These four MAGs have been classified within the phylum Firmicutes and had an activity profile (based on the global abundance variation of their transcripts over time) with a peak in the early stages of composting (day 3 to 15) (Supplementary Table 6).

Evidence for dissimilatory sulfite reductase function (dsr $\mathrm{AB}$ and aps $\mathrm{A})$ was not detected in the genomes, suggesting that the respiratory sulfate reduction was not the main strategy for anaerobic respiration employed by the MAGs here studied.

Table 2 Metabolic profile of ZC4 MAGs. ARGs: Antibiotic Resistance Genes

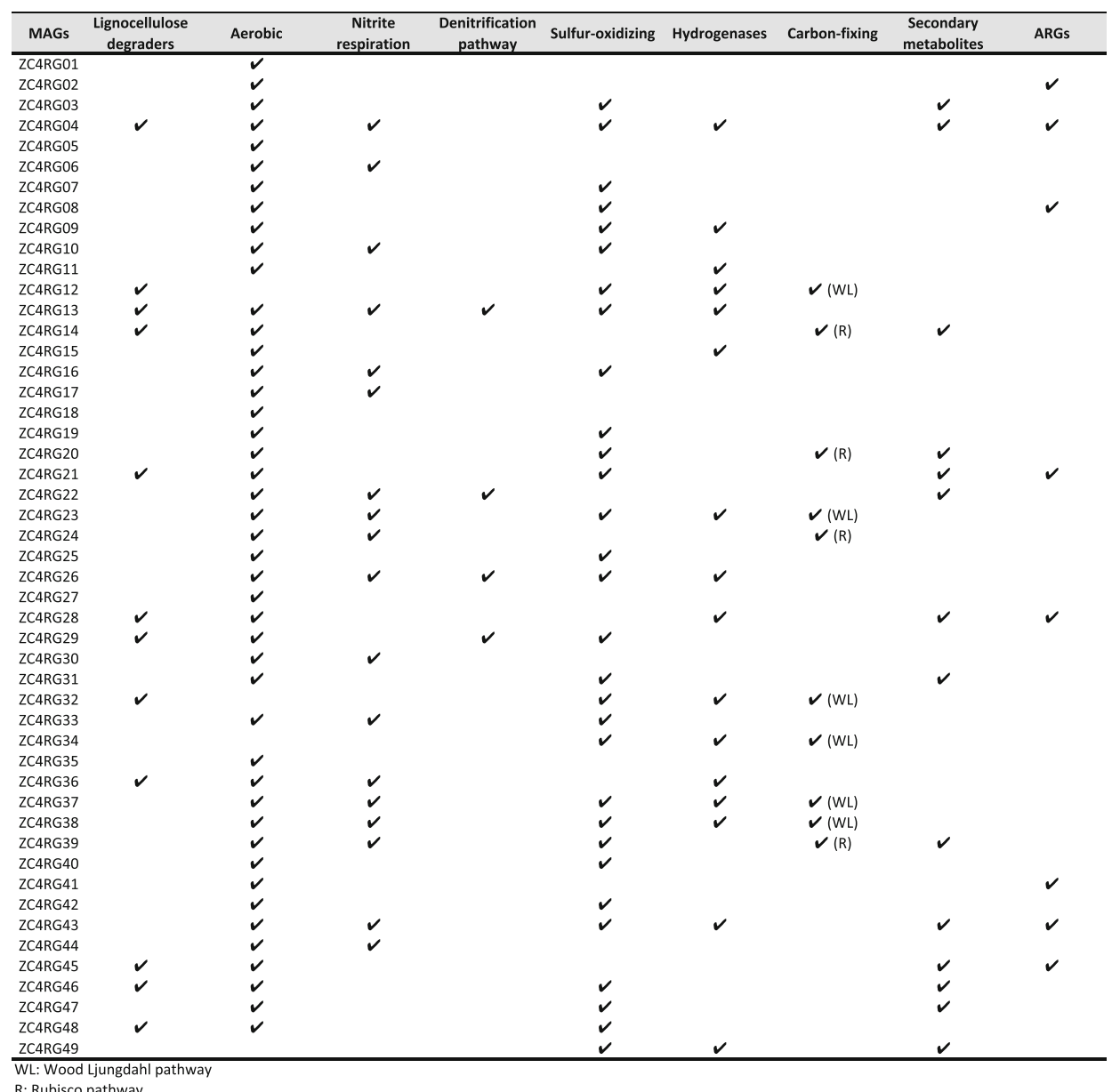

R: Rubisco pathway 
Active denitrification genes were detected in several MAGs (Table 2). Eighteen of them presented the nitrite respiration gene nirK. The variant $n i r \mathrm{~S}$ was not detected in the MAGs. ZC4RG13 (Rhodothermus marinus), ZC4RG22 (Luteitaleales), ZC4RG26 (Sphaerobacter thermophilus), and ZC4RG29 (Cyclobacteriaceae) encode the complete denitrification pathway (i.e., nitrous-oxide reductase pathway, nosZD). These MAGs also have genes that code for nirB instead of nirK for nitrite reductase (Table 2). Nitrous-oxide reductase genes (nos $\mathrm{ZD}$ ) were also active during the composting process. The variation in abundance of transcripts of these denitrification genes increased over time, with a peak in day 7 (Supplementary Fig. 2).

\section{Chemolithotrophic metabolism}

We found evidence for chemolithotrophic metabolism based on MAG genes related to the oxidation of inorganic sulfur compounds (Table 2). Nearly all MAGs have genes annotated with products from the sulfur oxidation pathway via sulfur dioxygenase. Some of the MAGs have genes annotated with other functions associated with the oxidation of sulfur compounds. ZC4RG20 (Gammaproteobacteria), for instance, represents a bacterial population that showed transcripts associated with sulfur dioxygenase, sulfide oxidation (sqr), and thiosulfate oxidation ( $\operatorname{sox} \mathrm{C})$, including transcripts associated with carbon fixation via rubisco activation, supporting a chemolithoautotrophic growth. The Sox system, which is able to oxidize sulfite and sulfone group in thiosulfate, was found in MAGs ZC4RG25 (Hyphomicrobiaceae), ZC4RG31 (Hyphomicrobiaceae), ZC4RG33 (Aquamicrobium), and ZC4RG42 (Betaproteobacteria), although soxC was apparently lacking in all of them. Sulfide oxidation (sulfide- quinone reductase, sqr) to thiosulfate was detected also in ZC4RG25. These observations highlight that members of the bacterial populations in the composting microbiome were capable of harvesting energy by oxidizing inorganic sulfur compounds. Coding sequences annotated as nitrification genes (amo and hao) were not detected in the ZC4 MAGs, indicating that this trait was of minor or no relevance for the composting microbiome.

Several hydrogenases were found to be present and expressed (Table 2, Supplementary Fig. 2). We were able to identify two types of hydrogenases. MAGs ZC4RG04, 09, 13, $15,26,28,36,37,38,43$, and 49 , belonging to diverse phyla (Table 1), have genes annotated as [NiFe] hydrogenases. MAGs ZC4RG11, 12, 23, 32, 34, 38, and 49, from the phylum Firmicutes (Table 1), have genes annotated as prototypical hydrogen-evolving [FeFe] hydrogenases (group A1).

\section{Correlation of MAGs based on their activity profiles}

Using the 49 ZC4 MAGs, we computed correlation patterns using their variation in activity during the composting process inferred from ZC4 metatranscriptomic data. The correlation patterns derived from the activity profile resulted in a graph composed by 43 nodes and 76 interactions, and three clusters (Fig. 5). In what follows we describe the main features of each activity cluster, highlighting days when a relatively high number of transcripts associated with specific MAGs in each cluster was observed (Supplementary Table 6).

\section{Cluster 1}

Seven MAGs form this cluster. Transcripts from members of this cluster are more abundant on initial days of composting (days 1 and 3) with a slight later increase on day 64 (immediately after turning), followed by another increase on day 99. Cluster members ZC4RG02 (Pseudoxanthomonas), ZC4RG04 (Thermobispora bispora), and ZC4RG28 (Streptosporangiaceae) presented the highest number of transcripts in the initial days.

\section{Cluster 2}

This cluster is composed of 10 MAGs, all Firmicutes and mostly abundant and active between days 3 and 15, followed by a peak on day 64. Many transcripts from ZC4RG12 (Caldicoprobacter) and ZC4RG32 (C. oshimai) related to lignocellulose degradation were identified, especially on days 3,7 , and 15 .

\section{Cluster 3}

This 26-MAG cluster is taxonomically diverse (it contains members of Acidobacteriota, Actinobacteriota, Bacteroidota, Chloroflexota, and Proteobacteria phyla). The following cluster members are notable for expressing genes related to lignocellulose breakdown: ZC4RG13 (Rhodothermus marinus), ZC4RG14 (Steroidobacteraceae), ZC4RG21 (Thermobifida fusca), ZC4RG26 (Sphaerobacter thermophilus), ZC4RG29 (Cyclobacteriaceae), ZC4RG36 (Anaerolinea), ZC4RG46 (Polyangiales), ZC4RG47 (Micromonospora), and ZC4RG48 (Roseiflexaceae); this activity is especially intense on days 30,78 , and 99. ZC4RG20 (Gammaproteobacteria) and ZC4RG45 (Thermocrispum agreste) express several genes associated with lignin degradation (i.e., annotated with CAZy AA families), especially on day 99.

\section{Metabolic dependencies based on genome-scale models}

Based on the results obtained with the correlation analysis using the transcriptional activity profile of MAGs (Fig. 5, Supplementary Table 6, Supplementary Table 12) and the activity of relevant genes (Table 2, Supplementary Tables 9, 10, 11, Fig. 3, and Supplementary Fig. 2), we identified MAGs according to their importance in the different stages of composting and the main functions associated with them (Fig. 6). We then used this framework to assess the metabolic dependencies 


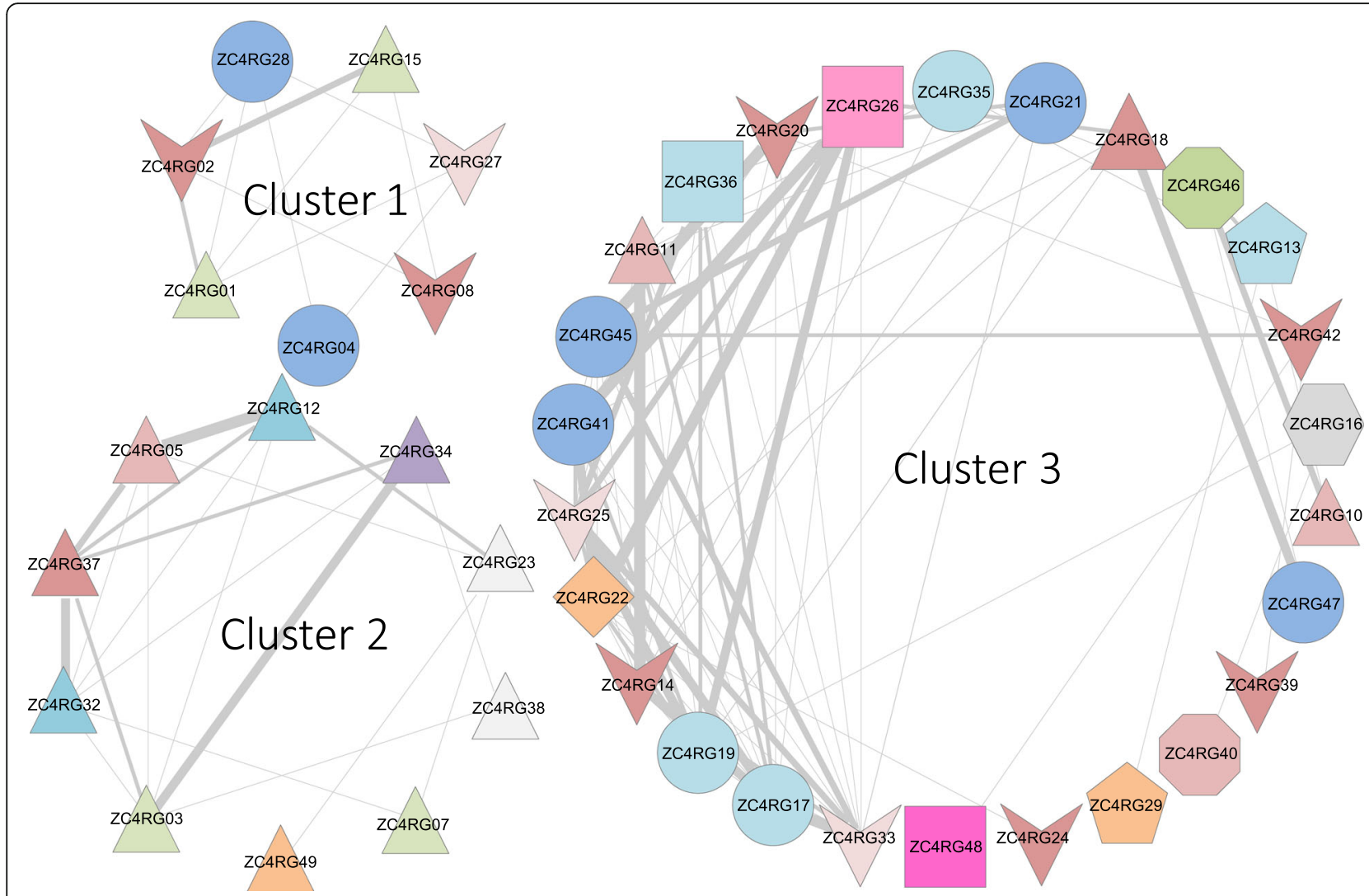

Fig. 5 MAGs co-occurrence based on their relative abundance in metatranscriptomes of the ZC4 composting cell. Nodes represent MAGs and edges represent Spearman correlations $\left(r^{2} \geq 0.8\right)$, in the following five intervals: $\geq 0.97 ;(0.97 . .0 .95] ;$ (0.95..0.92]; (0.92..0.90], and less than 0.90. (For actual pairwise values, see Supplementary Table 12.) Different shapes indicate different phyla: Acidobacteriaota (diamond), Actinobacteriota (circle), Bacteroidota (pentagon), Chloroflexota (square), Firmicutes (triangle), Gemmatimonadota (hexagon), Myxococcota (octagon), and Proteobacteria (inverted triangle). Colors indicate different classes within a phylum (see Table 1)

between these MAGs based on genome-scale models. The results revealed strong dependencies (i.e. the maximum dependency score $=1$ ) between some of the MAGs (Supplementary Table 13). According to the models obtained, the most frequent compounds involved in the interactions between MAGs are $\mathrm{H}^{+}, \mathrm{NH}_{3}, \mathrm{O}_{2}$, $\mathrm{CO}_{2}$, as well as glutamate, fumarate, succinate, glucose, and hypoxanthine (Supplementary Table 13). The top three MAGs with highest number of interactions for metabolic exchange were ZC4RG13 (Rhodothermus marinus), ZC4RG04 (Thermobispora bispora) and ZC4RG20 (Gammaproteobacteria). ZC4RG13 interactions were mostly as a compound donor, while ZC4RG04 and ZC4RG20 interactions were mostly as compound receivers (Supplementary Table 14A).

To test if the predicted metabolic interactions are likely to be specific of the bacterial strains that were able to thrive in the composting microbiome, we reran the metabolic interaction analysis replacing the MAGs assigned to known species by the respective reference genomes from GenBank according to the taxonomic assignment (Table 1). We found that ZC4RG04, ZC4RG13 and ZC4RG20 were no longer classified among the top three genomes with highest number of interactions in the model (Supplementary Table 14B).

\section{Discussion}

Sixty MAGs were recovered, of which 47 are potentially from new bacterial species and 24 represent novel genomes. Thirty-three of our MAGs had good matches to GEM MAGs (30) or to isolates (3) from environments in geographical areas distinct from where we got our samples. This fact shows that these MAGs (and by extrapolation, all MAGs in our dataset) are not artifacts, and are good approximations (as all MAGs are) to the genomes of the bacterial species living in these environments. It also suggests that similar environments, even if geographically very distant, have a tendency to select the same bacterial species. There may be some species that are specific to the environment we studied, but this will need to be rechecked in the future, as more MAGs from different parts of the world become available. 


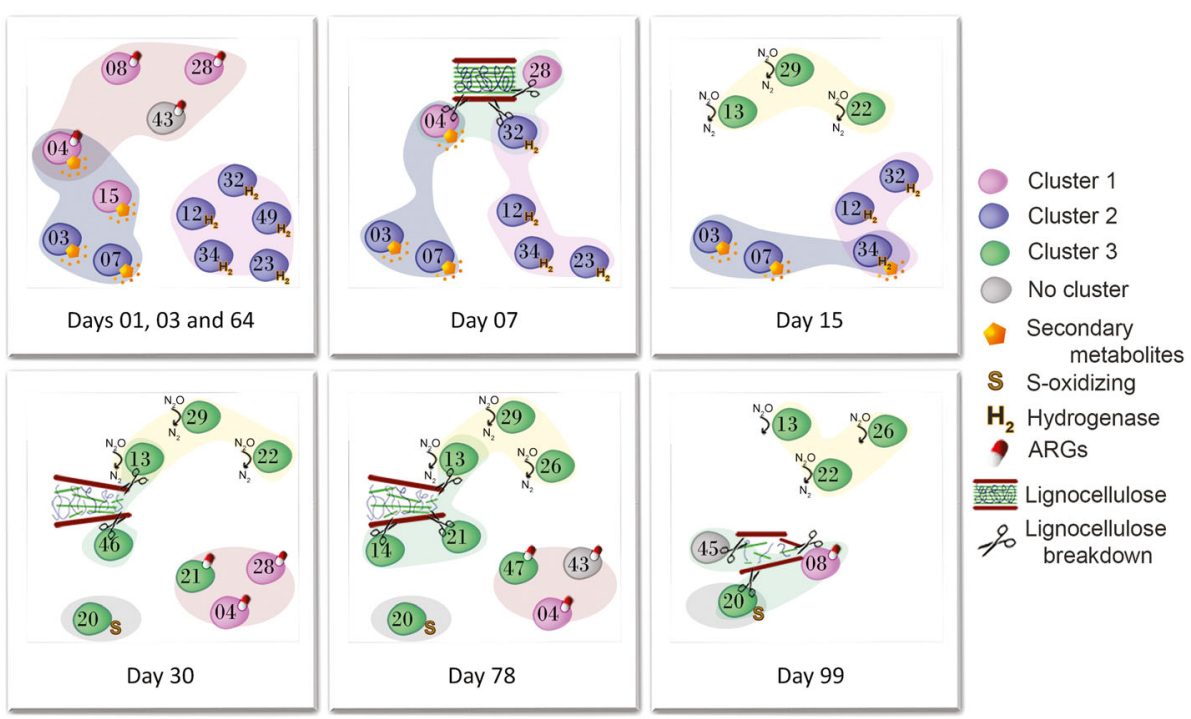

Fig. 6 Schematic representation of keystone microbial players according to their importance in the different stages of composting. MAGs are represented as roughly circular numbered shapes, and their colors reflect the cluster they were assigned to (Fig. 5 and Supplementary Table 4). Lignocellulose breakdown and relevant active functions are represented across the stages by the various symbols, and the irregular background shapes connect MAGs that express the same functions. The turning procedure was performed on day 63 , therefore day 64 is considered a recapitulation of the start of composting (days 1 and 3), based on the patterns of microbial functions and activity that we observed in the present study and in our previous work [1]

We focused our functional analysis on the 49 MAGs that were retrieved from the ZC4 composting cell. One may ask to what extent these 49 MAGs are representative of the composting process. We can answer this question by referring to our previous results, based on the same baseline datasets, and that took into account all shotgun sequencing reads [1]. At the phylum level, that work stated that Firmicutes, Proteobacteria, Bacteroidota and Actinobacteriota accounted for at least $85 \%$ of all classified reads in all samples. A breakdown of the 49 ZC4 MAGs in terms of phylum shows that those four phyla account for $86 \%$ of these 49 MAGs, therefore in close agreement with the previous result. At deeper taxonomic levels, the comparison becomes more difficult, primarily because read-based classification is still unreliable [30, 31]. On the other hand, the $49 \mathrm{ZC} 4$ MAGs account for $21.8 \%$ of all metagenome reads. Based on these results, we believe that the MAG-based functional results we present are representative of key microbial processes taking place in this thermophilic composting, while certainly very far from exhausting the tremendous functional diversity of this environment.

Among the recovered genomes, ZC4RG01 (Caldibacillus debilis), ZC4RG04 (Thermobispora bispora), ZC4RG13 (Rhodothermus marinus), ZC4RG21 (Thermobifida fusca), ZC4RG26 (Sphaerobacter thermophilus), ZC4RG32 (C. oshimai), and ZC4RG49 ([Clostridium] cellulosi) have been classified as species previously reported as being capable of cellulose degradation [13, 32-
37]. Two Chloroflexota MAGs, ZC4RG36 (Anaerolineae) and ZC4RG48 (Roseiflexaceae), are additional lignocellulose degraders that we have found, having many genes classified as CAZymes (326 and 313, respectively), exceeding the number of CAZymes in the much betterknown lignocellulose degraders Thermobispora bispora [38] and Thermobifida fusca [35], corresponding to ZC4RG04 and ZC4RG21, with 174 and 150 CAZymes, respectively. Chloroflexota bacteria have been reported in biomass-degrading environments using cultivationindependent methods, in some cases associated with the maturing phase of the composting process [39].

We focused our functional analysis on the following processes: lignocellulose degradation, denitrification, sulfur metabolism, hydrogen metabolism, oxygen metabolism, and secondary metabolite and antibiotics production. The need to focus on lignocellulose degradation is obvious given the nature of the samples. For the other processes, our justification is as follows. Denitrification: it is a facultative respiratory biochemistry pathway mediated by denitrifying microbes, which convert nitrate to nitrogen gas or nitrous oxide under strict anaerobic conditions, and constitutes one of the main branches of nitrogen cycle during composting [40]. Sulfur metabolism and hydrogen metabolism: during the thermogenic phase, degradation and mineralization of complex organic matter also take place by autotrophic sulfur oxidizers, which oxidize (and, thus, detoxify) the hydrogen sulfide generated by the mineralization of 
organic sulfur compounds, whereas hydrogen-oxidizing bacteria use the molecular hydrogen produced by fermentative reactions [41]. Oxygen metabolism: organic matter is composted both aerobically and anaerobically, but aerobic composting is the most efficient form of decomposition and produces finished compost in the shortest time. During the initial stages of composting, the oxidation of organic material by microbial populations, which increases temperature, is at its most intense, but this form of degradation goes on essentially all the time. Finally, secondary metabolite production and antibiotic resistance genes were also analyzed, given their crucial role in microbial interactions, which to a large extent drive the whole process.

We carried out a correlation analysis of MAGs based on their activity profiles during the process. Based on all these results, we propose here a framework for the temporal dynamics of microbial processes in the composting system we have studied (Fig. 6).

MAGs from clusters 1 and 2 (Fig. 5) are the main constituents of the composting stage characterized by days 1, 3 (composting start), and 64 (recapitulation of composting start after the turning procedure) (Fig. 6). These MAGs represent bacterial populations expressing ARG genes and genes with functions related to secondary metabolite production. These activities could be explained by intense competition between microorganisms. Indeed, these stages have high microbial diversity [1]. According to this interpretation, secondary metabolite production and ARG expression would be the consequence of the arms-shields race hypothesized to take place in the composting microbial community [42, 43]. Among the MAGs in these clusters, ZC4RG03 (Calditerricola) and ZC4RG07 (Bacillus), both from cluster 2, stand out in terms of expression of secondary metabolite genes between day 1 and day 15, and on day 64 (Supplementary Table 10). At a more detailed level, ZC4RG03 contains three adjacent highly expressed genes that are orthologous to the first three genes of the Plantaricin $\mathrm{C}$ operon in Lactobacillus plantarum [44]. Plantaricin $C$ has been shown to inhibit Gram-positive bacteria [45]. Based on this analysis, we hypothesize that ZC4RG03 and ZC4RG07 represent important players in the composting process, by producing relatively high amounts of compounds with selective antimicrobial activity against pathogenic and opportunistic competitor bacteria. At the same time, they also have the genes required to consume easily degradable compounds, which we assume are particularly abundant during the initial composting stages (or right after the turning procedure). A considerable portion of the composting substrate material that was sampled is made of animal feces, and niche protection through antagonistic competition has been observed in gut microbiota [46, 47].
According to our framework (Fig. 6), MAGs from cluster 2 are primarily active between days 1 and 15 (and right after turning) (Supplementary Table 6). All of them are Firmicutes encoding hydrogenases. $\mathrm{H}_{2}$ oxidizing bacteria are a group of facultative autotrophs that can use the molecular hydrogen produced during fermentative conversion of organic compounds as an electron donor during transitions from aerobic to anaerobic decomposition, which possibly justifies the relevance of MAGs with hydrogenase activity at this stage. Hydrogenases participate in the mechanism that allows bacteria to store metabolic energy as an electrochemical potential across the membrane via the proton-motive force. $\mathrm{H}_{2}$ metabolism can be coupled with $\mathrm{CO}_{2}$ as electron acceptor, which allows autotrophic growth via the acetyl coenzyme A (i.e., the Wood pathway) [48], supporting the metabolisms of diverse prokaryotes, including methanogens, aerobic carboxidotrophs, acetogens, sulfate-reducers, and hydrogenogenic bacteria. MAGs ZC4RG12, 32, 34 are in this last group of bacteria as, besides the hydrogenases, they possess the carbon monoxide dehydrogenase (codh gene). Methanogens and sulfate reducers were not detected among the recovered MAGs. Thus, although predominantly aerobic, our results suggest that, at this stage of the composting process (between days 1 and 15), microaerophilic habitats favor anaerobic fermentation.

The presence of obligately and facultatively sulfurand hydrogen-oxidizing bacteria was already reported in hot composts, suggesting that they may play a part in mineralization, and particularly in inorganic sulfur compound oxidation during the thermogenic phase $\left(>60^{\circ}\right)$ of the composting process [41]. Also, in the present work, sulfur oxidation capability was detected in ZC4RG25, ZC4RG31, ZC4RG33 and ZC4RG42. Together, these observations suggest that degradation and mineralization of complex organic matter takes place.

The late stages (represented by days 30, 78 and 99) are mainly dominated by MAGs from cluster 3 that perform lignocellulose degradation, denitrification, and sulfur oxidation. In these stages there is a decrease in the overall phylogenetic microbial diversity [1]. Nevertheless, cluster 3 contains the largest and most diverse group of MAGs. We hypothesize that in these stages most nutrients derive from recalcitrant material (e.g., lignin). ZC4RG20 (Gammaproteobacteria) is a cluster 3 MAG with a large repertoire of enzymes classified as Auxiliary Activities, when compared to other MAGs. One MAG that does not belong to cluster 3 but nevertheless seems to be particularly active around day 99 is ZC4RG08 (Pseudomonas thermotolerans) (cluster 1). It is the 
only one to have genes annotated as belonging to the AA1 family. AA1 enzymes that have been experimentally studied are multicopper oxidases that use diphenols and related substances as donors, with oxygen as acceptor, and are known for their role in the enzymatic conversion of recalcitrant polysaccharides such as lignin [27]. ZC4RG08 is also noteworthy because it is the MAG that expresses the largest variety of ARGs among the MAGs here studied, suggesting it may be intrinsically resistant to several antibiotics; this characteristic has been reported for other members of the Pseudomonas genus [49].

Other noteworthy cluster 3 MAGs are ZC4RG13 (Rhodothermus marinus), ZC4RG21 (Thermobifida fusca), ZC4RG22 (Luteitaleales), and ZC4RG29 (Cyclobacteriaceae) (Fig. 5). During the late stages it is hypothesized that oxygen becomes more limited inside the composting pile and denitrification processes come into play (Supplementary Fig. 2). Accordingly, the above MAGs, plus ZC4RG26 (Sphaerobacter thermophilus), not included in any cluster, express genes annotated as nitrous-oxide reductase (including nos $\mathrm{Z}$ and nos $\mathrm{D}$ ), which is the last step in denitrification, thus being evidence that these MAGs are able to perform the complete pathway (Table 2). The ability to utilize nitrous oxide $\left(\mathrm{N}_{2} \mathrm{O}\right)$ for anaerobic respiration might be crucial to improve efficiency of nitrogen utilization by bacteria in the composting process. Moreover, it is worth mentioning that $\mathrm{N}_{2} \mathrm{O}$ is a potent greenhouse gas, and microbial conversion of $\mathrm{N}_{2} \mathrm{O}$ to $\mathrm{N}_{2}$ is so far the unique sink known for $\mathrm{N}_{2} \mathrm{O}$ in the biosphere [50]. Anaerobic respiration using nitrous oxide is a widespread trait in prokaryotes, however not all denitrifiers encode this final step in denitrification [50]. As mentioned, one of the MAGs expressing nitrous oxide reductase is ZC4RG22, which was classified as a member of the Acidobacteriota phylum. To our knowledge this is the first report of a nitrous oxide reductase in this phylum [50].

By applying metabolic modeling methods, we were able to predict metabolic dependencies between MAGs. These results suggest that metabolic interactions in composting can be determined by complementary functions found in the genomes of producers and consumers. According to the Black Queen hypothesis [51], in order to increase fitness, one microbial population may lose genes related to a function when that function is already provided by another microbial population in the community. Therefore, the genomic differences between closely related strains are likely to be driven by local adaptation and coevolutionary interactions [51, 52]. This could explain why composting bacteria can present different metabolic dependencies compared with closely related strains that were isolated from other environments (Supplementary Table 14). Based on the predictions of compounds exchanged more frequently, our model suggests that major interactions were related to fermentation products (Supplementary Table 13). Dependencies on $\mathrm{H}^{+}$exchange can be associated with hydrogenase activity (Table 2) and proton flux across membranes, which is related to ATP synthesis, $\mathrm{pH}$ homeostasis, and maintenance of solute gradients [53].

A metabolite transported into the extracellular environment as a waste product by one bacterium is often used by neighboring bacteria $[51,52]$. This could also explain the $\mathrm{O}_{2}$ exchange flux identified (Supplementary Table 13). Oxygen can be a product derived from reactive oxygen species (ROS) detoxification systems, such as those encoded by chlorate-reducing bacteria via chloride dismutase [54]. Due to intense redox activity, ROSdetoxification is a vital function in the composting microbiome, as observed also by the overall profile of dominant functions detected in the ZC4 metatranscriptome dataset (Supplementary Table 15). Succinate also plays important roles in ROS management [55]. The higher exchange rates of succinate can also be associated with a potential demand for succinylation of cellulase enzymes, since it has been demonstrated that this process can enhance enzyme activity nearly twofold [56]. Thus, our data suggest a putative positive feedback loop in the composting microbiome, by which leak sugars from cellulose breakdown are shared from degraders to fermenters, and then fermenters return fermentation byproducts such as succinate to degraders, and this can increase efficiency of cellulolytic enzymes. Another important detected pathway is related to the phosphate starvation response (Supplementary Table 15), which is also consistent with the dependency of phosphate exchange between MAGs (Supplementary Table 13).

Our model shows that ZC4RG13 (Rhodothermus marinus), ZC4RG04 (Thermobispora bispora), and ZC4RG20 (a novel Gammaproteobacteria species) are potential key players in the metabolic interactions during lignocellulose saccharification process (Supplementary Table 14). Although their genomes were recovered only from ZC4 metagenomes, our stringent genome recovery criteria may have prevented the recovery of these genomes in the datasets from the ZC3 composting cell. We do have evidence that these genomes were also present in the ZC3 composting metagenome (Supplementary Table 16).

Among the hypotheses generated in this work that could be verified experimentally, we mention the possible isolation of the Gammaproteobacteria bacterium (ZC4RG20), which presents the largest repertoire of AAs enzymes among analyzed MAGs, as well as the isolation of the ZC4 strains of Thermobispora bispora, Rhodothermus marinus, and fermentative bacteria that encode hydrogenases and are capable of exchanging fermentation 
by-products. These isolates could be tried as biomass degrading bacterial inoculants, and they might be particularly promising if they encode novel pathways towards efficient saccharification on industrial processes.

\section{Conclusions}

With this work, we have added new knowledge on various aspects of the São Paulo Zoo composting process, on top of our previous work [1]. We now have solid hypotheses as to which bacteria are the main players in the process, and know almost all of their gene contents. Twenty-four of these bacteria are completely novel. We were able to get an idea of the temporal variation in abundance of each of these organisms throughout the composting process. This in turn enabled us to build a dynamic model of interactions of these bacteria, which is also a contribution to the molecular understanding of composting in general. The model, of course, is a hypothesis, and additional research will be needed to verify its predictions. Taken together, our results contribute to future research aiming at the engineering of efficient biomass-degrading thermophilic microbiomes.

\section{Methods}

\section{Composting metagenomic and metatranscriptomic data}

The composting metagenomic datasets on which this study is based have been described previously [1]. Briefly, the samples come from the composting facility at the São Paulo Zoo Park in the city of São Paulo, Brazil. Two composting cells were sampled: one called ZC3 and the other ZC4. For both, composting lasted 99 days at $60-70{ }^{\circ} \mathrm{C}$ most of the time. For ZC3, samples were collected on days $1,30,64,78$, and 99 , and for ZC4 they were collected on days $1,3,7,15,30,64,67,78$, and 99 of the thermophilic composting process. A turning procedure was performed on day 65 for ZC3 and on day 63 for ZC4. DNA shotgun sequencing was done for all samples, and metatranscriptome sequencing was done for all ZC4 samples except day 64 sample [1].

\section{MAGs recovery workflow}

Shotgun metagenomic reads from all samples from ZC3 and ZC4 composting cells were filtered and softtrimmed (Quality values 212 ) using BBDuk from the BBTools package version 37.96 (https://jgi.doe.gov/dataand-tools/bbtools/). All shotgun reads used in the assemblies were obtained in a previous work [1]. Reads with length shorter than $80 \mathrm{bp}$ were removed and the remaining reads were de novo assembled using metaSpades $3.12(\mathrm{k}$-mer $=21,33,55,77,99,113,121,127$, --meta $)$ [57] (Supplementary Table 17). To obtain MAGs, the following steps were carried out, for each composting cell (ZC3 and ZC4) (Supplementary Fig. 3): 1) reads from all samples were assembled and the contigs binned with Metabat2 version 2.11 [58]; 2) reads from individual samples were assembled and the corresponding contigs were binned using Metabat2. After these two steps, only bins with completeness at least $50 \%$ and contamination at most $10 \%$ were kept for further processing, based on results from CheckM 1.0.12 [18]; 3) bins from individual samples (step 2) were compared with each other using Mash 2.1 [59], which allowed us to establish when the "same" bin occurred on different days (Mash distance at most 0.05$)$; 4) for each "distinct" bin determined in step 3 its reads were reassembled and the results rebinned with Metabat2 [58] and MyCC version 1 [60]; 5) bins from step (1) and those from step (4) were compared, again using Mash; 6) the MAGs selected for additional analyses were those distinct bins with best completeness and contamination results (when there was more than one bin for the same MAG), provided completeness was at least $80 \%$ and contamination at most $11 \%$ (Supplementary Fig. 3).

\section{Taxonomic assignment and genome comparisons}

MAG taxonomic assignment was based on GTDB [26]. For those assignments that reached the species level, we carried out further comparisons with reference genomes of those species (whenever available, with complete genomes). These and other comparisons were done with the ANI tool (http://enve-omics.ce.gatech.edu/ani/) and with GGDC [61]. We refer to MAGs by their identifiers, providing in parenthesis the GTDB classification according to phylum, class, order, family, genus, or species. The comparison between our MAGs and those of the GEM catalog [22] was done with the tool fastANI 1.1 [62]. The GEM catalog makes available files with metadata information (the file is called genome_metadata, available in sql and tsv formats). These can be found at https://portal.nersc.gov/GEM/genomes/.

\section{Functional annotation}

MAGs were annotated using the NCBI Prokaryotic Genome Annotation Pipeline [63]. Their protein-coding gene sequences were compared against the Clusters of Orthologous Groups (COGs) [64] database using rpsblast+ (blast version 2.9.0) [65], with a cut-off e-value of at most $10^{-5}$. COG categories were assigned to the best hits with cdd2cog script (https://github.com/ aleimba/bac-genomics-scripts/tree/master/cdd2cog). The amino acid sequences of the predicted coding sequences were classified for carbohydrate-active enzymes (CAZymes) [27] using dbCAN2 [66] using parameter values as described in http://bcb.unl.edu/dbCAN2/help. php. In the CAZymes database, enzymes are categorized in different classes and families, including key enzymes for lignocellulose degradation such as glycoside hydrolases (GHs) and auxiliary activities (AA), and the 
following complementary enzymes: glycosyltransferases (GTs), polysaccharide lyases (PLs), carbohydrate esterases (CEs) and carbohydrate-binding modules (CBMs). Genes were also classified using a set of HMMs for detecting other metabolic pathways of interest, such as genes involved in denitrification, sulfur metabolism, hydrogen metabolism, and oxygen metabolism [24, 67]. Evidence supporting MAGs with strictly anaerobic metabolism was obtained based on the consistency between the results provided by TRAITAR and gene classification as oxidases [68]. The global profile of functions in the metatranscriptomic dataset was determined using FMAP release v0.15 [69].

The presence of antibiotic resistance genes was analyzed in the MAGs by comparing protein-coding sequences against the CARD database (April 2019) [70] using the Resistance Gene Identifier via the CARD website RGI portal. We filtered out all results below 70\% identity and $85 \%$ sequence coverage. antiSMASH 5.0 was used to find gene clusters involved in the biosynthesis of secondary metabolites [71].

\section{Abundance and activity profiles of MAGs}

The activity profile of MAGs across the metatranscriptomic datasets was obtained using the function quant bins provided by metaWRAP 1.3 followed by normalization based on TPM (transcripts per kilobase million) [21]. Similarly, relative abundance of expressed genes was obtained by determining metatranscriptome reads that mapped to coding sequences using BEDTools 2.27.1 [72], followed by normalization based on TPM. We use the term transcripts to refer to MAG coding sequences to which metatranscriptome reads could be mapped.

\section{Correlation of MAGs based on their activity profiles}

To identify patterns of co-occurring bacteria represented by MAGs in the ZC4 datasets, correlation analysis was performed based on relative abundance of MAGs and their transcripts, as described. We used CONET 1.1.1.beta [73] (Spearman $\mathrm{r}^{2}>0.8$ ) and the resulting graphs were visualized in Cytoscape 3.2.1 [74].

\section{Metabolic interaction models}

Based on MAG co-occurrence patterns and the relative abundance of transcripts with annotation related to biomass degradation, denitrification, sulfur metabolism, hydrogen metabolism, and oxygen metabolism, we defined subsets of MAGs according to their importance in the different stages of the composting process. For each subset we built a metabolic interaction model using SMETANA 1.1.0 [75], based on genome-scale metabolic reconstructions that were obtained from files annotated in the PATRIC platform [76]. The results were submitted to Kbase [77] in order to run the Build Metabolic Model function, including the default option gapfilling, which relies on the ModelSEED Biochemistry Database [78]. With this method, metabolic genes were mapped onto biochemical reactions, and this information was integrated with information on stoichiometry reactions, subcellular localization, biomass composition, and estimation of thermodynamic feasibility, in order to produce a detailed stoichiometric model of metabolism at the genome scale. Metabolic dependency score calculated by SMETANA is normalized to a range between 0 and 1, meaning complete independency and complete dependency, respectively. Only strong metabolic dependencies; i.e., score $=1$ [75] were considered.

\section{Abbreviations}

AA: Auxiliary Activities; ANI: Average Nucleotide Identity; ARG: Antibiotic Resistance Gene; CAZy: Carbohydrate-Active enZYmes Database; CARD: Comprehensive Antibiotic Resistance Database; CBM: CarbohydrateBinding Module; CE: Carbohydrate Esterase; COG: Cluster of Orthologous Group; DDH: DNA-to-DNA hybridization; GH: Glycoside Hydrolase;

GT: Glycosyltransferase; HE: Highly Expressed; HMM: Hidden Markov Model; MAG: Metagenome-Assembled Genome; NCBI: National Center for Biotechnology Information; PL: Polysaccharide Lyase; TPM: Transcripts per kilobase million reads

\section{Supplementary Information}

The online version contains supplementary material available at https://doi. org/10.1186/s12864-021-07957-9.

\section{Additional file 1. \\ Additional file 2.}

\section{Acknowledgements}

We are grateful to Paulo Magalhães Bressan and João Batista da Cruz (Fundação Parque Zoológico de São Paulo) for providing access to the composting facility. We also thank Carlos Morais Piroupo for computational support.

Authors' contributions

JCS and AMDS conceived the study. JCS, LPPB, RVP, LFM, and LMSM performed the analyses. FBS and JSLP helped with the comparative and phylogenetic analyses of the MAGs. JCS and AMDS wrote the final manuscript with contributions from LFM, LPPB, RVP, and LMSM. All authors have read, revised, and approved the final manuscript.

\section{Funding}

Funding for this research was provided by São Paulo Research Foundation (FAPESP), research grant 11/50870-6 and by the Coordination for the Improvement of Higher Education Personnel (CAPES), research grant 3385/ 2013. LPPB was supported by FAPESP fellowship 18/19247-0. LMSM and RVP were supported by fellowships from CAPES. AMDS and JCS are supported in part by Research Fellowship Awards from the National Council for Scientific and Technological Development (CNPq). The funders had no role in study design, data collection, analysis, decision to publish or preparation of the manuscript.

\section{Availability of data and materials}

The genome sequence and annotation of all MAGs described in this work are available from GenBank, and their accession numbers and permanent links are the following: 


\begin{tabular}{|c|c|c|c|}
\hline MAG_ID & BioSample & Accession & Link \\
\hline ZC3RG01 & SAMN08097825 & PITW00000000 & $\begin{array}{l}\text { https://www.ncbi.nlm.nih.gov/ } \\
\text { nuccore/PITW00000000 }\end{array}$ \\
\hline ZC3RG02 & SAMN08097829 & PITX00000000 & $\begin{array}{l}\text { https://www.ncbi.nlm.nih.gov/ } \\
\text { nuccore/PITX00000000 }\end{array}$ \\
\hline ZC3RG03 & SAMN08097823 & PITV00000000 & $\begin{array}{l}\text { https://www.ncbi.nlm.nih.gov/ } \\
\text { nuccore/PITV00000000 }\end{array}$ \\
\hline ZC3RG04 & SAMN08096851 & PITU00000000 & $\begin{array}{l}\text { https://www.ncbi.nlm.nih.gov/ } \\
\text { nuccore/PITU00000000 }\end{array}$ \\
\hline ZC3RG05 & SAMN08101807 & PITY00000000 & $\begin{array}{l}\text { https://www.ncbi.nlm.nih.gov/ } \\
\text { nuccore/PITY00000000 }\end{array}$ \\
\hline ZC3RG06 & SAMN08101822 & PITZ00000000 & $\begin{array}{l}\text { https://www.ncbi.nlm.nih.gov/ } \\
\text { nuccore/PITZ00000000 }\end{array}$ \\
\hline
\end{tabular}

ZC3RG07 SAMN08101823 PIUA00000000 https://www.ncbi.nlm.nih.gov/

ZC3RG08 SAMN08102237 PIUB00000000 https://www.ncbi.nlm.nih.gov/ nuccore/PIUB00000000

ZC3RG09 SAMN09092868 QGSS00000000 https://www.ncbi.nlm.nih.gov/ nuccore/QGSS00000000

ZC3RG10 SAMN09092869 QGSR00000000 https://www.ncbi.nlm.nih.gov/ nuccore/QGSR00000000

ZC3RG11 SAMN09092870 QGSQ00000000 https://www.ncbi.nlm.nih.gov/ nuccore/QGSQ00000000

ZC4RG01 SAMN08227010 PKQW00000000 https://www.ncbi.nlm.nih.gov/ nuccore/PKQW00000000

ZC4RG02 SAMN08227009 PKQV00000000 https://www.ncbi.nlm.nih.gov/ nuccore/PKQV00000000

ZC4RG03 SAMN08227008 PKQU00000000 https://www.ncbi.nlm.nih.gov/ nuccore/PKQU00000000

ZC4RG04 SAMN08227007 PKQT00000000 https://www.ncbi.nlm.nih.gov/ nuccore/PKQT00000000

ZC4RG05 SAMN08227006 PKQS00000000 https://www.ncbi.nlm.nih.gov/ nuccore/PKQS00000000

ZC4RG06 SAMN08227005 PKQR00000000 https://www.ncbinlm.nih.gov/ nuccore/PKQR00000000

ZC4RG07 SAMN08227012 PKQY00000000 https://www.ncbi.nlm.nih.gov/ nuccore/PKQY00000000

ZC4RG08 SAMN08227013 PKQZ00000000 https://www.ncbi.nlm.nih.gov/ nuccore/PKQZ00000000

ZC4RG09 SAMN08227011 PKQX00000000 https://www.ncbi.nlm.nih.gov/ nuccore/PKQX00000000

ZC4RG10 SAMN08227015 PKRB00000000 https://www.ncbi.nlm.nih.gov/ nuccore/PKRB00000000

ZC4RG11 SAMN08227014 PKRA00000000 https://www.ncbi.nlm.nih.gov/ nuccore/PKRA00000000

ZC4RG12 SAMN08227016 PKRC00000000 https://www.ncbi.nlm.nih.gov/ nuccore/PKRC00000000

ZC4RG13 SAMN08227017 PKRD000000000 https://www.ncbi.nlm.nih.gov/ nuccore/PKRD00000000

ZC4RG14 SAMN08227018 PKRE00000000 https://www.ncbi.nlm.nih.gov/ nuccore/PKRE00000000

ZC4RG15 SAMN08227019 PKRF00000000 https://www.ncbi.nlm.nih.gov/ nuccore/PKRF00000000

ZC4RG16 SAMN09092792 QGVH00000000 https://www.ncbi.nlm.nih.gov/ nuccore/QGVH00000000
(Continued)

\begin{tabular}{llll}
\hline MAG_ID & BioSample & Accession & Link \\
\hline ZC4RG17 & SAMN08227020 & PKRG00000000 & https:/www.ncbi.nlm.nih.gov/ \\
& nuccore/PKRG00000000 \\
ZC4RG18 SAMN08227022 & PKRI00000000 & $\begin{array}{l}\text { https://www.ncbi.nlm.nih.gov/ } \\
\text { nuccore/PKRI00000000 }\end{array}$ \\
ZC4RG19 SAMN08227021 & PKRH00000000 & https://www.ncbi.nlm.nih.gov/ \\
& nuccore/PKRH00000000
\end{tabular}

ZC4RG20 SAMN08227023 PKRJ00000000 https://www.ncbi.nlm.nih.gov/ nuccore/PKRJ00000000

ZC4RG21 SAMN09092793 QGVG00000000 https://www.ncbi.nlm.nih.gov/ nuccore/QGVG00000000

ZC4RG22 SAMN09092794 QGVF00000000 https://www.ncbi.nlm.nih.gov/ nuccore/QGVF00000000

ZC4RG23 SAMN09092795 QGVE00000000 https://www.ncbi.nlm.nih.gov/ nuccore/QGVE00000000

ZC4RG24 SAMN09092796 QGVD00000000 https://www.ncbi.nlm.nih.gov/ nuccore/QGVD00000000

ZC4RG25 SAMN09092797 QGVC00000000 https://www.ncbi.nlm.nih.gov/ nuccore/QGVC00000000

ZC4RG26 SAMN09092798 QGVB00000000 https://www.ncbi.nlm.nih.gov/ nuccore/QGVB00000000

ZC4RG27 SAMN09092799 QGVA00000000 https://www.ncbi.nlm.nih.gov/ nuccore/QGVA00000000

ZC4RG28 SAMN09092800 QGUZ00000000 https://www.ncbi.nlm.nih.gov/ nuccore/QGUZ00000000

ZC4RG29 SAMN09092801 QGUY00000000 https://www.ncbi.nlm.nih.gov/ nuccore/QGUY00000000

ZC4RG30 SAMN09092802 QGUX00000000 https://www.ncbi.nlm.nih.gov/ nuccore/QGUX00000000

ZC4RG31 SAMN09092803 QGUW00000000 https://www.ncbi.nlm.nih.gov/ nuccore/QGUW00000000

ZC4RG32 SAMN09092804 QGUV00000000 https://www.ncbi.nlm.nih.gov/ nuccore/QGUV00000000

ZC4RG33 SAMN09092805 QGUU00000000 https://www.ncbi.nlm.nih.gov/ nuccore/QGUU00000000

ZC4RG34 SAMN09092806 QGUT00000000 https://www.ncbi.nlm.nih.gov/ nuccore/QGUT00000000

ZC4RG35 SAMN09092807 QGUS00000000 https://www.ncbi.nlm.nih.gov/ nuccore/OGUS00000000

ZC4RG36 SAMN09092808 QGUR00000000 https://www.ncbi.nlm.nih.gov/ nuccore/QGUR00000000

ZC4RG37 SAMN09092809 QGUQ00000000 https://www.ncbi.nlm.nih.gov/ nuccore/QGUQ00000000

ZC4RG38 SAMN09092810 QGUP00000000 https://www.ncbi.nlm.nih.gov/ nuccore/QGUP00000000

ZC4RG39 SAMN09092811 QGU000000000 https://www.ncbi.nlm.nih.gov/ nuccore/QGUO00000000

ZC4RG40 SAMN09092812 QGUN00000000 https://www.ncbi.nlm.nih.gov/ nuccore/QGUN00000000

ZC4RG41 SAMN09092813 QGUM00000000 https://www.ncbi.nlm.nih.gov/ nuccore/QGUM00000000

ZC4RG42 SAMN09092814 QGUL00000000 https://www.ncbi.nlm.nih.gov/ nuccore/QGUL00000000

ZC4RG43 SAMN09092815 QGUK00000000 https://www.ncbi.nlm.nih.gov/ nuccore/QGUK00000000 
(Continued)

\begin{tabular}{|c|c|c|c|}
\hline MAG_ID & BioSample & Accession & Link \\
\hline ZC4RG44 & SAMN09092816 & QGUJ00000000 & $\begin{array}{l}\text { https://www.ncbi.nlm.nih.gov/ } \\
\text { nuccore/QGUJ00000000 }\end{array}$ \\
\hline ZC4RG45 & SAMN09092817 & QGUI00000000 & $\begin{array}{l}\text { https://www.ncbi.nlm.nih.gov/ } \\
\text { nuccore/QGUI00000000 }\end{array}$ \\
\hline ZC4RG46 & SAMN09092818 & QGUH00000000 & $\begin{array}{l}\text { https://www.ncbi.nlm.nih.gov/ } \\
\text { nuccore/QGUH00000000 }\end{array}$ \\
\hline ZC4RG47 & SAMN09092819 & QGUG00000000 & $\begin{array}{l}\text { https://www.ncbi.nlm.nih.gov/ } \\
\text { nuccore/QGUG00000000 }\end{array}$ \\
\hline ZC4RG48 & SAMN09092820 & QGUF00000000 & $\begin{array}{l}\text { https://www.ncbi.nlm.nih.gov/ } \\
\text { nuccore/QGUF00000000 }\end{array}$ \\
\hline ZC4RG49 & SAMN09092821 & QGUE00000000 & $\begin{array}{l}\text { https://www.ncbi.nlm.nih.gov/ } \\
\text { nuccore/QGUEO0000000 }\end{array}$ \\
\hline
\end{tabular}

The datasets supporting the conclusions of this article are included within the article (and its additional files).

\section{Declarations}

Ethics approval and consent to participate

Not applicable.

\section{Consent for publication}

Not applicable.

\section{Competing interests}

The authors declare that they have no competing interests.

\section{Author details}

${ }^{1}$ Departamento de Bioquímica, Instituto de Química, Universidade de São Paulo, São Paulo, Brazil. ²Programa de Pós-Graduação Interunidades em Bioinformática, Universidade de São Paulo, São Paulo, Brazil. ${ }^{3}$ Laboratório Especial de Ciclo Celular, Instituto Butantan, São Paulo, SP, Brazil.

Received: 19 March 2021 Accepted: 23 August 2021 Published online: 10 September 2021

\section{References}

1. Antunes LP, Martins LF, Pereira RV, Thomas AM, Barbosa D, Lemos LN, et al. Microbial community structure and dynamics in thermophilic composting viewed through metagenomics and metatranscriptomics. Sci Rep. 2016;6: ARTN 38915. https://doi.org/10.1038/srep38915.

2. Palaniveloo K, Amran MA, Norhashim NA, Mohamad-Fauzi N, Peng-Hui F, Hui-Wen $L$, et al. Food waste composting and microbial community structure profiling. Processes. 2020;8(6). https://doi.org/10.3390/pr8060723.

3. Jurado MM, Camelo-Castillo AJ, Suarez-Estrella F, Lopez MJ, Lopez-Gonzalez JA, Estrella-Gonzalez MJ, et al. Integral approach using bacterial microbiome to stabilize municipal solid waste. J Environ Manage. 2020;265:110528. https://doi.org/10.1016/j.jenvman.2020.110528.

4. Ma L, Zhao Y, Meng L, Wang X, Yi Y, Shan Y, et al. Isolation of Thermostable Lignocellulosic Bacteria From Chicken Manure Compost and a M42 Family Endocellulase Cloning From Geobacillus thermodenitrificans Y7. Front Microbiol. 2020;11:ARTN 281. https://doi.org/10.3389/fmicb.2020.00281.

5. Zhong X-Z, Li X-X, Zeng Y, Wang S-P, Sun Z-Y, Tang Y-Q. Dynamic change of bacterial community during dairy manure composting process revealed by high-throughput sequencing and advanced bioinformatics tools. Bioresour Technol. 2020;306:123091. https://doi.org/10.1016/j.biortech.202 0.123091 .

6. Kolinko S, Wu YW, Tachea F, Denzel E, Hiras J, Gabriel R, et al. A bacterial pioneer produces cellulase complexes that persist through community succession. Nat Microbiol. 2018;3(1). https://doi.org/10.1038/s41564-0170052-z.

7. Montella S, Ventorino V, Lombard V, Henrissat B, Pepe O, Faraco V. Discovery of genes coding for carbohydrate-active enzyme by metagenomic analysis of lignocellulosic biomasses. Sci Rep. 2017;7:ARTN 42623. https://doi.org/10.1038/srep42623.

8. Jimenez DJ, Dini-Andreote F, DeAngelis KM, Singer SW, Salles JF, van Elsas JD. Ecological insights into the dynamics of plant biomass-degrading microbial consortia. Trends Microbiol. 2017;25(10):788-96. https://doi.org/1 0.1016/j.tim.2017.05.012

9. Lindemann SR, Bernstein HC, Song HS, Fredrickson JK, Fields MW, Shou WY, et al. Engineering microbial consortia for controllable outputs. ISME J. 2016; 10(9):2077-84. https://doi.org/10.1038/ismej.2016.26.

10. Silva NM, de Oliveira A, Pegorin S, Giusti CE, Ferrari VB, Barbosa D, et al. Characterization of novel hydrocarbon-degrading Gordonia paraffinivorans and Gordonia sihwensis strains isolated from composting. PLoS One. 2019; 14(4):16. https://doi.org/10.1371/journal.pone.0215396.

11. Lutz S, Thuerig B, Oberhaensli T, Mayerhofer J, Fuchs JG, Widmer F, et al. Harnessing the microbiomes of suppressive composts for plant protection: from metagenomes to beneficial microorganisms and reliable diagnostics. Front Microbiol. 2020;11. https://doi.org/10.3389/fmicb.2020.01810.

12. Viikari $L$, Vehmaanpera J, Koivula A. Lignocellulosic ethanol: from science to industry. Biomass Bioenergy. 2012;46:13-24. https://doi.org/10.1016/j. biombioe.2012.05.008

13. Lemos LN, Pereira RV, Quaggio RB, Martins LF, Moura LMS, da Silva AR, et al. Genome-centric analysis of a thermophilic and cellulolytic bacterial consortium derived from composting. Front Microbiol. 2017;8:644. https:// doi.org/10.3389/fmicb.2017.00644.

14. Mello BL, Alessi AM, Riano-Pachon DM, de Azevedo ER, FEG G, MCE S, et al. Targeted metatranscriptomics of compost-derived consortia reveals a GH11 exerting an unusual exo-1,4-beta-xylanase activity. Biotechnol Biofuels. 2017; 10. https://doi.org/10.1186/s13068-017-0944-4

15. Eloe-Fadrosh EA, Paez-Espino D, Jarett J, Dunfield PF, Hedlund BP, Dekas AE, et al. Global metagenomic survey reveals a new bacterial candidate phylum in geothermal springs. Nat Commun. 2016;7(1):10. https://doi.org/10.1038/ ncomms 10476

16. Martins LF, Antunes LP, Pascon RC, Franco de Oliveira JC, Digiampietri LA, Barbosa D, et al. Metagenomic analysis of a tropical composting operation at the Sao Paulo Zoo Park reveals diversity of biomass degradation functions and organisms. PLoS One. 2013:8(4). https://doi.org/10.1371/ journal.pone.0061928.

17. Liang Z, Shi J, Wang C, Li J, Liang D, Yong EL, et al. Genome-centric metagenomic insights into the impact of alkaline/acid and thermal sludge pretreatment on the microbiome in digestion sludge. Appl Environ Microbiol. 2020;86(23):e01920. https://doi.org/10.1128/AEM.0192 $0-20$.

18. Parks DH, Imelfort M, Skennerton CT, Hugenholtz P, Tyson GW. CheckM: assessing the quality of microbial genomes recovered from isolates, single cells, and metagenomes. Genome Res. 2015;25(7):1043-55. https://doi.org/1 $0.1101 /$ gr.186072.114.

19. Sangwan N, Xia FF, Gilbert JA. Recovering complete and draft population genomes from metagenome datasets. Microbiome. 2016;4:ARTN 8. https:// doi.org/10.1186/s40168-016-0154-5.

20. Parks DH, Rinke C, Chuvochina M, Chaumeil PA, Woodcroft B, Evans PN, et al. Recovery of nearly 8,000 metagenome-assembled genomes substantially expands the tree of life. Nat Microbiol. 2018;3(2):253. https:// doi.org/10.1038/s41564-017-0083-5.

21. Uritskiy GV, DiRuggiero J, Taylor J. MetaWRAP-a flexible pipeline for genome-resolved metagenomic data analysis. Microbiome. 2018;6:ARTN 158. https://doi.org/10.1186/s40168-018-0541-1.

22. Nayfach S, Roux S, Seshadri R, Udwary D, Varghese N, Schulz F, et al. A genomic catalog of Earth's microbiomes. Nat Biotechnol. 2020;39(4):499509. https://doi.org/10.1038/s41587-020-0718-6.

23. Bandla A, Pavagadhi S, Sudarshan AS, Poh MCH, Swarup S. 910 metagenome-assembled genomes from the phytobiomes of three urbanfarmed leafy Asian greens. Sci Data. 2020;7(1):ARTN 278. https://doi.org/10.1 038/s41597-020-00617-9

24. Anantharaman K, Brown CT, Hug LA, Sharon I, Castelle CJ, Probst AJ, et al. Thousands of microbial genomes shed light on interconnected biogeochemical processes in an aquifer system. Nat Commun. 2016;7:ARTN 13219. https://doi.org/10.1038/ncomms13219.

25. Bowers RM, Kyrpides NC, Stepanauskas R, Harmon-Smith M, Doud D, Reddy TBK, et al. Minimum information about a single amplified genome (MISAG) and a metagenome-assembled genome (MIMAG) of bacteria and archaea. Nat Biotechnol. 2017;35(8):725-31. https://doi.org/10.1038/nbt.3893. 
26. Chaumeil PA, Mussig AJ, Hugenholtz P, Parks DH. GTDB-Tk: a toolkit to classify genomes with the genome taxonomy database. Bioinformatics. 2020;36(6):1925-7. https://doi.org/10.1093/bioinformatics/btz848.

27. Lombard V, Ramulu HG, Drula E, Coutinho PM, Henrissat B. The carbohydrate-active enzymes database (CAZy) in 2013. Nucleic Acids Res. 2014;42(D1):D490-5. https://doi.org/10.1093/nar/gkt1178.

28. Goldstein BP. Resistance to rifampicin: a review. J Antibiot. 2014;67(9):62530. https://doi.org/10.1038/ja.2014.107.

29. Zalucki YM, Dhulipala V, Shafer WM. Dueling regulatory properties of a transcriptional activator (MtrA) and repressor (MtrR) that control efflux pump gene expression in Neisseria gonorrhoeae. Mbio. 2012;3(6):e00446-12. https://doi.org/10.1128/mBio.00446-12.

30. Mclntyre ABR, Ounit R, Afshinnekoo E, Prill RJ, Hénaff E, Alexander N, et al. Comprehensive benchmarking and ensemble approaches for metagenomic classifiers. Genome Biol. 2017;18(1):182. https://doi.org/10.1186/s13059-01 7-1299-7.

31. Tran Q, Phan V. Assembling reads improves taxonomic classification of species. Genes-Basel. 2020;11(8):ARTN 946. https://doi.org/10.3390/genes11 080946

32. Bjornsdottir SH, Blondal T, Hreggvidsson GO, Eggertsson G, Petursdottir S, Hjorleifsdottir $S$, et al. Rhodothermus marinus: physiology and molecular biology. Extremophiles. 2006;10(1):1-16. https://doi.org/10.1007/s00792-0050466-z.

33. Yokoyama H, Wagner ID, Wiegel J. Caldicoprobacter oshimai gen. Nov., sp nov., an anaerobic, xylanolytic, extremely thermophilic bacterium isolated from sheep faeces, and proposal of Caldicoprobacteraceae fam. nov. Int J Syst Evol Microbiol. 2010;60(1):67-71. https://doi.org/10.1099/ijs.0.011379-0.

34. Zhang KD, Chen XH, Schwarz WH, Li FL. Synergism of glycoside hydrolase Secretomes from two thermophilic bacteria cocultivated on lignocellulose. Appl Environ Microbiol. 2014;80(8):2592-601. https://doi.org/10.1128/Aem. 00295-14.

35. del Pulgar EMG, Saadeddin A. The cellulolytic system of Thermobifida fusca. Crit Rev Microbiol. 2014;40(3):236-47. https://doi.org/10.3109/1040841x.2013. 776512

36. Wang C, Dong D, Wang H, Mueller $K$, Qin $Y$, Wang $H$, et al. Metagenomic analysis of microbial consortia enriched from compost: new insights into the role of Actinobacteria in lignocellulose decomposition. Biotechnol Biofuels. 2016;9. https://doi.org/10.1186/s13068-016-0440-2.

37. Hiras J, Wu YW, Deng K, Nicora CD, Aldrich JT, Frey D, et al. Comparative community proteomics demonstrates the unexpected importance of actinobacterial glycoside hydrolase family 12 protein for crystalline cellulose hydrolysis. Mbio. 2016;7(4):ARTN e01106. https://doi.org/10.1128/mBio.011 06-16.

38. Liolios K, Sikorski J, Jando M, Lapidus A, Copeland A, Glavina T, et al. Complete genome sequence of Thermobispora bispora type strain (R51). Stand Genomic Sci. 2010;2(3):318-26. https://doi.org/10.4056/sigs.962171.

39. Puentes-Tellez PE, Salles JF. Construction of effective minimal active microbial consortia for lignocellulose degradation. Microb Ecol. 2018;76(2): 419-29. https://doi.org/10.1007/s00248-017-1141-5.

40. Shi M, Zhao Y, Zhu L, Song X, Tang Y, Qi H, et al. Denitrification during composting: Biochemistry, implication and perspective. Int Biodeter Biodegr. 2020;153(105043). https://doi.org/10.1016/j.ibiod.2020.105043.

41. Beffa T, Blanc M, Aragno M. Obligately and facultatively autotrophic, sulfurand hydrogen-oxidizing thermophilic bacteria isolated from hot composts. Arch Microbiol. 1996;165(1):34-40. https://doi.org/10.1007/s002030050293.

42. D'Costa VM, Griffiths E, Wright GD. Expanding the soil antibiotic resistome: exploring environmental diversity. Curr Opin Microbiol. 2007;10(5):481-9. https://doi.org/10.1016/j.mib.2007.08.009.

43. Nesme J, Simonet P. The soil resistome: a critical review on antibiotic resistance origins, ecology and dissemination potential in telluric bacteria. Environ Microbiol. 2015;17(4):913-30. https://doi.org/10.1111/1462-2920.12631.

44. Florez AB, Mayo B. Genome analysis of lactobacillus plantarum LL441 and genetic characterisation of the locus for the Lantibiotic Plantaricin C. Front Microbiol. 2018:9:1916. https://doi.org/10.3389/fmicb.2018.01916.

45. Gonzalez B, Glaasker E, Kunji E, Driessen A, Suarez JE, Konings WN. Bactericidal mode of action of plantaricin C. Appl Environ Microbiol. 1996; 62(8):2701-9. https://doi.org/10.1128/AEM.62.8.2701-2709.1996.

46. Garcia-Gutierrez E, Mayer MJ, Cotter PD, Narbad A. Gut microbiota as a source of novel antimicrobials. Gut Microbes. 2019;10(1):1-21. https://doi. org/10.1080/19490976.2018.1455790.
47. lacob S, lacob DG, Luminos LM. Intestinal microbiota as a host defense mechanism to infectious threats. Front Microbiol. 2019:9:ARTN 3328. https:// doi.org/10.3389/fmicb.2018.03328.

48. Drake HL, Daniel SL, Kusel K, Matthies C, Kuhner C, Braus-Stromeyer S. Acetogenic bacteria: what are the in situ consequences of their diverse metabolic versatilities? Biofactors. 1997;6(1):13-24. https://doi.org/10.1002/ biof.5520060103.

49. Blair JMA, Webber MA, Baylay AJ, Ogbolu DO, Piddock LJV. Molecular mechanisms of antibiotic resistance. Nat Rev Microbiol. 2015;13(1):42-51. https://doi.org/10.1038/nrmicro3380.

50. Hallin S, Philippot L, Loffler FE, Sanford RA, Jones CM. Genomics and ecology of novel N2O-reducing microorganisms. Trends Microbiol. 2018; 26(1):43-55. https://doi.org/10.1016/j.tim.2017.07.003.

51. Morris JJ, Lenski RE, Zinser ER. The black queen hypothesis: evolution of dependencies through adaptive gene loss. Mbio. 2012;3(2):ARTN e00036-12. https://doi.org/10.1128/mBio.00036-12.

52. Thompson JN. Relentless evolution. Chicago: University of Chicago Press; 2013. https://doi.org/10.7208/chicago/9780226018898.001.0001.

53. Kakinuma $Y$. Inorganic cation transport and energy transduction in enterococcus hirae and other streptococci. Microbiol Mol Biol R. 1998;62(4): 1021-45. https://doi.org/10.1128/Mmbr.62.4.1021-1045.1998.

54. Ettwig KF, Speth DR, Reimann J, Wu ML, Jetten MSM, Keltjens JT. Bacterial oxygen production in the dark. Bba-Bioenergetics. 1817;2012:S155. https:// doi.org/10.1016/j.bbabio.2012.06.406.

55. Lemire J, Alhasawi A, Appanna VP, Tharmalingam S, Appanna VD. Metabolic defence against oxidative stress: the road less travelled so far. J Appl Microbiol. 2017;123(4):798-809. https://doi.org/10.1111/jam.13509.

56. Selkala T, Sirvio JA, Lorite GS, Liimatainen H. Anionically stabilized cellulose Nanofibrils through Succinylation pretreatment in urea-Lithium chloride deep eutectic solvent. Chemsuschem. 2016;9(21):3074-83. https://doi.org/1 $0.1002 /$ cssc.201600903

57. Nurk S, Meleshko D, Korobeynikov A, Pevzner PA. metaSPAdes: a new versatile metagenomic assembler. Genome Res. 2017;27(5):824-34. https:// doi.org/10.1101/gr.213959.116

58. Kang DWD, Li F, Kirton E, Thomas A, Egan R, An H, et al. MetaBAT 2: an adaptive binning algorithm for robust and efficient genome reconstruction from metagenome assemblies. Peerj. 2019;7:ARTN e7359. https://doi.org/10. 7717/peerj.7359.

59. Ondov BD, Treangen TJ, Melsted P, Mallonee AB, Bergman NH, Koren S, et al. Mash: fast genome and metagenome distance estimation using MinHash. Genome Biol. 2016;17:ARTN 132. https://doi.org/10.1186/s13059-01 6-0997-x.

60. Lin HH, Liao YC. Accurate binning of metagenomic contigs via automated clustering sequences using information of genomic signatures and marker genes. Sci Rep. 2016;6:ARTN 24175. https://doi.org/10.1038/srep24175.

61. Meier-Kolthoff JP, Auch AF, Klenk HP, Goker M. Genome sequence-based species delimitation with confidence intervals and improved distance functions. Bmc Bioinformatics. 2013;14:ARTN 60. https://doi.org/10.1186/14 71-2105-14-60.

62. Jain C, Rodriguez RL, Phillippy AM, Konstantinidis KT, Aluru S. High throughput ANI analysis of 90K prokaryotic genomes reveals clear species boundaries. Nat Commun. 2018;9(1):5114. https://doi.org/10.1038/s41467-01 8-07641-9.

63. Tatusova T, DiCuccio M, Badretdin A, Chetvernin V, Nawrocki EP, Zaslavsky L, et al. NCBI prokaryotic genome annotation pipeline. Nucleic Acids Res. 2016 44(14):6614-24. https://doi.org/10.1093/nar/gkw569.

64. Galperin MY, Makarova KS, Wolf YI, Koonin EV. Expanded microbial genome coverage and improved protein family annotation in the COG database. Nucleic Acids Res. 2015;43(D1):D261-9. https://doi.org/10.1093/ nar/gku1223.

65. Camacho C, Coulouris G, Avagyan V, Ma N, Papadopoulos J, Bealer K, et al. BLAST plus : architecture and applications. Bmc Bioinformatics. 2009;10: ARTN 421. https://doi.org/10.1186/1471-2105-10-421.

66. Zhang $H$, Yohe $T$, Huang $L$, Entwistle $S$, Wu P, Yang Z, et al. dbCAN2: a meta server for automated carbohydrate-active enzyme annotation. Nucleic Acids Res. 2018:46(W1):W95-W101. https://doi.org/10.1093/nar/gky418.

67. Carnevali PBM, Schulz F, Castelle CJ, Kantor RS, Shih PM, Sharon I, et al. Hydrogen-based metabolism as an ancestral trait in lineages sibling to the Cyanobacteria. Nat Commun. 2019;10(463):ARTN 1451. https://doi.org/10.103 8/s41467-019-09423-3 
68. Weimann A, Mooren K, Frank J, Pope PB, Bremges A, McHardy AC. From genomes to phenotypes: traitar, the microbial trait analyzer. Msystems. 2016;1(6):ARTN e00101. https://doi.org/10.1128/mSystems.00101-16.

69. Kim JW, Kim MS, Koh AY, Xie Y, Zhan XW. FMAP: functional mapping and analysis pipeline for metagenomics and metatranscriptomics studies. Bmc Bioinformatics. 2016;17:ARTN 420. https://doi.org/10.1186/s12859-016-1278-0.

70. Alcock BP, Raphenya AR, Lau TTY, Tsang KK, Bouchard M, Edalatmand A, et al. CARD 2020: antibiotic resistome surveillance with the comprehensive antibiotic resistance database. Nucleic Acids Res. 2020;48(D1):D517-25. https://doi.org/10.1093/nar/gkz935.

71. Medema MH, Blin K, Cimermancic $\mathrm{P}$, de Jager V, Zakrzewski $\mathrm{P}$, Fischbach MA, et al. antiSMASH: rapid identification, annotation and analysis of secondary metabolite biosynthesis gene clusters in bacterial and fungal genome sequences. Nucleic Acids Res. 2011;39(suppl_2):W339-46. https:// doi.org/10.1093/nar/gkr466

72. Quinlan AR, Hall IM. BEDTools: a flexible suite of utilities for comparing genomic features. Bioinformatics. 2010;26(6):841-2. https://doi.org/10.1093/ bioinformatics/btq033.

73. Faust K, Raes J. CoNet app: inference of biological association networks using Cytoscape. F1000Research. 2016. https://doi.org/10.12688/f1000resea rch.9050.2.

74. Shannon P, Markiel A, Ozier O, Baliga NS, Wang JT, Ramage D, et al. Cytoscape: a software environment for integrated models of biomolecular interaction networks. Genome Res. 2003;13(11):2498-504. https://doi.org/1 $0.1101 /$ gr.1239303.

75. Zelezniak A, Andrejev S, Ponomarova O, Mende DR, Bork P, Patil KR. Metabolic dependencies drive species co-occurrence in diverse microbial communities. P Natl Acad Sci USA. 2015;112(51):-E7156. https://doi.org/10.1 073/pnas.1522642113.

76. Davis JJ, Wattam AR, Aziz RK, Brettin T, Butler R, Butler RM, et al. The PATRIC bioinformatics resource center: expanding data and analysis capabilities. Nucleic Acids Res. 2020;48(D1):D606-12. https://doi.org/10.1093/nar/gkz943.

77. Arkin AP, Cottingham RW, Henry CS, Harris NL, Stevens RL, Maslov S, et al. KBase: the United States Department of Energy Systems Biology Knowledgebase. Nat Biotechnol. 2018;36(7):566-9. https://doi.org/10.1038/ nbt.4163.

78. Henry CS, DeJongh M, Best AA, Frybarger PM, Linsay B, Stevens RL. Highthroughput generation, optimization and analysis of genome-scale metabolic models. Nat Biotechnol. 2010;28(9):977-U922. https://doi.org/10.1 038/nbt.1672.

\section{Publisher's Note}

Springer Nature remains neutral with regard to jurisdictional claims in published maps and institutional affiliations.

Ready to submit your research? Choose BMC and benefit from:

- fast, convenient online submission

- thorough peer review by experienced researchers in your field

- rapid publication on acceptance

- support for research data, including large and complex data types

- gold Open Access which fosters wider collaboration and increased citations

- maximum visibility for your research: over $100 \mathrm{M}$ website views per year

At BMC, research is always in progress.

Learn more biomedcentral.com/submissions 